\title{
Die Maxima der Auslösekurve der kosmischen Ultrastrahlschauer
}

\author{
Von H. ThurN* und W. Bothe \\ Aus dem I. Physikalischen Institut der Universität Heidelberg \\ und dem Institut für Physik im Max-Planck-Institut für medizinische Forschung \\ (Z. Naturforschg. 6a, 576-591 [1951]; eingegangen am 30. Juni 1951) \\ Erich Regener zum 70. Geburtstag
}

In Fortsetzung der Versuche von $\mathrm{S}$ ch me is e r und B oth e ${ }^{3-5}$ werden die Schauer des 2. und 3. Maximums der Schauerauslösekurve von Blei mit einer neuen Koinzidenz- und Antikoinzidenz-Anordnung genauer untersucht. Ergebnisse: Die Schauer entstehen nicht unmittelbar, sondern stufen- oder kaskadenartig aus einer Primärstrahlung, können jedoch keine Elektron-Photon-Kaskaden sein, weil sie hierfür zu eng und zu durchdringend sind; ihre Absorpticnslänge in Blei beträgt $\mathrm{rd} .100 \mathrm{~g} / \mathrm{cm}^{2}$. Ihre maximale Häufigkeit unter Blei ist höchstens um eine Größenordnung geringer als die der $\mu$-Mesonen. Die Schauer des 2. Maximums werden durch geladene Teilchen, vermutlich $\mu$-Mesonen, erzeugt und bestehen wahrscheinlich aus einem geladenen Teilchen (etwa dem auslösenden Meson) und einer Anzahl neutraler Teilchen von vorläufig unbekannter Art. Die Schauer des 3. Maximums werden durch eine neutrale Strahlung (möglicherweise neutrale $\mu$-Mesonen) erzeugt und enthalten wahrscheinlich zwei geladene (vermutlich Mesonen) und vielleicht noch eine Anzahl neutraler Teilchen. Die Lage des 3. Maximums hängt von dem auf Meeresniveau reduzierten Luftdruck in der Weise ab, daß eine Luftdruckzunahme von $1 \mathrm{~cm} \mathrm{Hg}$ das 3. Maximum um 1,6 cm Pb nach kleineren Dicken verschiebt. Mit anderen atmosphärischen Faktoren, auch solchen der oberen Atmosphäre, scheint ein Zusammenhang nur so weit zu bestehen, wie diese mit dem reduzierten Luftdruck zusammenhängen. Filter, die sich einige Meter über dem Schauerstrahler befinden, haben dagegen keinen Einfluß auf die Lage des 3. Maximums. Eine sichere Deutung hierfür kann noch nicht gegeben werden.

\section{Ziel der Untersuchung}

$S$ chaltet man zwei oder mehr nebeneinander liegende Zählrohre in Koinzidenz und bringt darüber eine Materieschicht an, so können die in der Schicht entstehenden Ultrastrahlschauer als Koinzidenzen nachgewiesen werden. Trägt man die Häufigkeit dieser Koinzidenzen als Funktion der Schichtdicke des Schauerstrahlers auf, so erhält man die „Auslösekurve“ oder „Rossi-Kurve“. Rossi fand, daß diese Kurve, z. B. mit Blei als Schauerstrahler, ein Maximum bei einer Bleidicke von $17-35 \mathrm{~g} / \mathrm{cm}^{2}$ hat, je nach den geometrischen Verhältnissen. Dieses Maximum hat seine vollständige Deutung gefunden in den aus Elektronen und Photonen bestehenden „Kaskadenschauern“.

Im Jahre 1934 fanden $\mathrm{A} \mathrm{c} \mathrm{k} \mathrm{e} \mathrm{m} \mathrm{a} \mathrm{n} \mathrm{n}{ }^{1}$ und $\mathrm{H} \mathrm{u} \mathrm{m}$ m e ${ }^{2}$, daß die Auslösekurve bei größeren Bleidicken $\left(\right.$ rd. $\left.180 \mathrm{~g} / \mathrm{cm}^{2}\right)$ ein zweites Maximum hat. Dieses

* Diss. Heidelberg.

1 M. A c k e m a n n, Naturwiss. 22, 169 [1934].

2 J. N. H u m me l, Naturwiss. 22, 170 [1934].

3 K. S chmeiser u. W. B othe, Ann. Physik 32, 161 [1938].

${ }_{4}^{4}$ K. S c h m e is e r, Z. Physik 112, 501 [1939]. wurde 1937-39 eingehender untersucht von S ch meiser und B o the $\mathrm{e}^{3-5}$, die dabei, auf Grund der damaligen Kenntnisse über die kosmische Ultrastrahlung, zu folgenden Schlüssen kamen. 1. Die für das 2. Maximum verantwortlichen Schauer bestehen nur aus wenigen ionisierenden Teilchen. 2. Die Schauerteilchen sind nicht Elektronen, sondern wahrscheinlich Mesonen. 3. Die Divergenzwinkel dieser Schauer sind klein, nämlich höchstens $10-15^{\circ} .4$. Die diese Schauer auslösende Strahlung ist überwiegend eine ionisierende und gehört zur harten Komponente der kosmischen Strahlung. 5. Der Wirkungsquerschnitt für die Schauererzeugung ist etwa proportional der Ordnungszahl $Z$ des Schauerstrahlers (während für die Schauer des 1. Maximums ungefähre Proportionalität mit $Z^{2}$ gilt).

Die Existenz des 2. Maximums wurde noch von anderen Seiten bestätigt ${ }^{6-16,16 a}$, während eine Anzahl

5 K. S c h m e is e r, Z. Physik 110, 443 [1938].

6 A. D rigo, Ric. sci. Progr. tecn. Econ. naz. 5, 88 [1934] und 6, 529 [1935]

7 H. M a a s, Ann. Physik 27, 507 [1936].

8 J. A. Pri e b s c h, S.-B. Akad. Wiss. Wien, Abt. II a 145, 101 [1936]. 
weiterer Autoren ${ }^{17-25}$ das 2. Maximum nicht finden konnten und für die positiven Ergebnisse der ersteren Autoren teilweise recht triviale Deutungen zu geben versuchten.

1936 wurde von $\mathrm{Priebsch}{ }^{8}$ noch ein 3. Maximum der Auslösekurve von Blei bei rd. $300 \mathrm{~g} / \mathrm{cm}^{2}$ beobachtet. Die Existenz des 3. Maximums wurde bestätigt von $\mathrm{Clay}$ und Mitarbeitern ${ }^{12,15}$ sowie von $\mathrm{Fenyves}$ und $\mathrm{Haiman}{ }^{26}$. Im einzelnen traten aber auch hier Unstimmigkeiten zwischen den Ergebnissen der verschiedenen Autoren auf. Genaueres über Entstehung und Eigenschaften der Schauer des 3. Maximums war bisher nicht bekannt.

Der Nachweis der Schauer des 2. und 3. Maximums ist im Prinzip auch ohne Koinzidenzen möglich, indem man mit einem einzigen Zählrohr die Absorptionskurve der Ultrastrahlung aufnimmt. Da die Treffwahrscheinlichkeit für einen Schauer größer ist als für ein Einzelteilchen, treten in der Absorptionskurve mehr oder weniger deutliche Buckel an den Stellen des 2. und 3. Maximums auf 7, 12, 26, 27.

Während das 1. Maximum keine wesentlichen Probleme mehr zu bieten scheint, sind die Prozesse, die zum 2. und 3. Maximum führen, noch völlig unbekannt. Deshalb, und in Anbetracht der teilweise widersprechenden Ergebnisse anderer Autoren, wurden die durch den Krieg unterbrochenen Koinzidenzuntersuchungen ${ }^{3-5}$ mit erheblich verbesserter Apparatur wieder aufgenommen und auf das 3. Maximum ausgedehnt. Der wesentliche Fortschritt besteht darin, daß die Ergiebigkeit der Anordnung, also die Häufigkeit der beobachteten Ereignisse, um mehr als eine Größenordnung gesteigert werden konnte. Dies brachte nicht nur eine viel höhere Meßgenauigkeit mit sich, sondern auch noch den Vorteil größerer Beweglichkeit, so daß der Einfluß rasch veränderlicher

9 J. C l ay, A. van Gemert u. J. T. Wiers ma, Physica 3, 627 [1936].

10 R. H. W o odward, Physic. Rev. 49, 711 [1936].

11 H. de Vos, Nature [London] 145, 387 [1940].

12 J. Clay, A. Venema u. K. H. Jonker, Physica 7, 673 [1940].

13 L. B roussard u. A. Graver, Physic. Rev. 60, 413 [1941].

14 M. Conversi, Physic. Rev. 70, 749 [1950].

15 J. Clay u. W. L. S cheen, Physica 14, 489 [1948].

16 J. Cla y u. G. K le in, Physica 16, 278 [1950]. [1951].

16a P. K. S en Cha d hury, Physic. Rev. 81, 274

17 A. S c hw e g le r, Z. Physik 101, 93 [1936].

18 W. M. Nielsen, J. E. M organ u. K. Z. M or g a n, Rev. mod. Physics 11, 287 [1939].

19 P. Auger, R. Maze, P.E hrenfest u. A. Freon, J. Physique Radium 10, 1 [1939]. äußerer Faktoren, z. B. des Luftdruckes, sicherer erfaßt werden konnte.

Im folgenden wird nur über reine Zählrohrmessungen berichtet. Ergänzende Untersuchungen nach anderen Methoden sind noch nicht abgeschlossen.
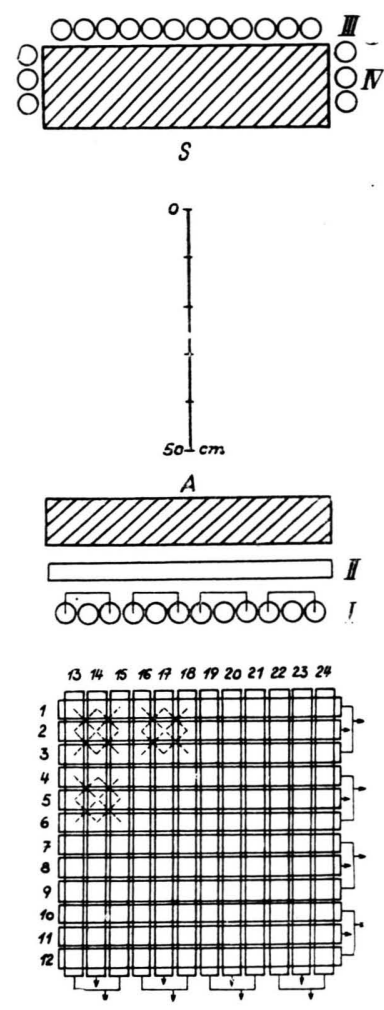

Abb. 1. Allgemeine Versuchsanordnung.

2. Allgemeine Versuchsanordnung (Abb. 1)

Ein verstrebtes Holzgestell aus vier Pfosten von $11 \times 11 \mathrm{~cm}$ und zwei Querbalken von $11 \times 15 \mathrm{~cm}$ trug auf vier $\mathrm{U}$ Eisenschienen $(4 \times 4 \times 4 \times 0,5 \mathrm{~cm})$ den Schauerstrahler $S$

20 W. M. Niels en, J. E. Morgan u. K. Z. M or g a n, Physic. Rev. 65, 995 [1939].

21 J. C l a y, Rev. mod. Physics 11, 281 [1939].

22 G. O. Altmann, H. N. Walker u. V.F.Heß, Physic. Rev. 58, 1011 [1940].

${ }_{23}$ E. P. George, J. Janossy u. M. M c Caig, Proc. Roy. Soc. [London], Ser. A 180, 219 [1942].

24 M. F orro u. Z. Ozorai, Naturwiss. 31, 140 [1943].

25 Z. O z o r a i, Z. Physik 122, 413 [1944].

26 E. F e $n$ yves u. O. Ha iman, Nature [London] 165, 244 [1950].

27 Dies gilt natürlich nicht mehr, wenn der Öffnungswinkel des Nachweisinstrumentes groß gegen denÖffnungswinkel der Schauer ist. Daher konnten R. G. H e y l a n d u. W. E. Duncanson, Nature [London] 167, 895 [1951], mit breiten Lagen parallelgeschalteter Zählrohre keine Buckel in der Absorptionskurve finden. 


\begin{tabular}{|l|c|c|c|c|c|}
\hline $\begin{array}{l}\text { Lage I } \\
\text { Lage II }\end{array}$ & $\begin{array}{c}1+3 \mid 2 \\
13+15 \mid 14\end{array}$ & $\begin{array}{c}1+3 \mid 2 \\
13+17 \mid 15\end{array}$ & $\begin{array}{c}1+5 \mid 3 \\
13+17 \mid 15\end{array}$ & $\begin{array}{c}1+5 \mid 3 \\
13+21 \mid 17\end{array}$ & $\begin{array}{c}1+9 \mid 5 \\
13+21 \mid 17\end{array}$ \\
\hline Mittl. Winkel & 3,4 & 5,3 & 6,8 & 10,7 & 12,7 \\
\hline
\end{tabular}

Tab. 1. Zählrohrgruppierungen.

aus Blei mit einer Fläche von $60 \times 60 \mathrm{~cm}$ und variabler Dicke. Unter dem Schauerstrahler waren zwei gekreuzte Zählrohrlagen von je 12 Zählrohren angebracht. Lage I hatte $100 \mathrm{~cm}$, Lage II $93 \mathrm{~cm}$ Abstand von der Unterseite des Schauerstrahlers. Die Messingzählrohre hatten $4 \mathrm{~cm}$ Innendurchmesser, $0,1 \mathrm{~cm}$ Wandstärke und 57 oder $60 \mathrm{~cm}$ Länge, die Füllung war 30 Torr Argon + 20 Torr Alkoholdampf. Der Achsenabstand benachbarter Zählrohre war $4,5 \mathrm{~cm}$. Die Ansprechwahrscheinlichkeit der Zählróhre wurde in der üblichen Weise zu 98,3\% bestimmt. Zwischen je zwei Zählrohren waren geerdete Abschirmbleche angebracht, um gegenseitige Influenzierung zu verhindern.

Das Meßprinzip sei an dem in Abb. 1 gezeichneten Fall erläutert. Im allgemeinen wurden ZweistrahlKoinzidenzen gemessen (Messung von DreistrahlKoinzidenzen s. Ziff. 12), und zwar in Form von Vierfach-Koinzidenzen zwischen je zwei Zählrohren der Lagen I und II. Die Bedingung für das Zustandekommen einer solchen Vierfachkoinzidenz ist offenbar, daß von den vier Flächenquadraten, die den oberen und unteren beiden Zählrohren gemeinsam sind, zwei diagonal gelegene gleichzeitig getroffen werden. Um nun die gesamte Zählfläche möglichst vollständig und mit möglichst geringem Aufwand an Verstärkern auszunutzen, wurden jeweils von drei äquidistanten Zählrohren einer Lage die beiden äußeren parallel und das mittlere damit in Koinzidenz geschaltet. Abb. 1 illustriert den übersichtlichsten Fall, bei dem die drei Zählrohre einer solchen Gruppe unmittelbar benachbart sind. Durch gestrichelte Linien sind die Quadratkombinationen angedeutet, die bei dieser Schaltung zur Wirkung kommen. Eine Koinzidenz zwischen einer solchen Quadratkombination zeigt einen Schauer von mindestens zwei Strahlen an, dessen Öffnungswinkel durch den Abstand der beiden Auftreffstellen voneinander und vom Schauerstrahler bestimmt ist. Man sieht, daß der Winkelbereich der so erfaßten Schauer nach oben eng begrenzt ist, im Gegensatz zu den älteren Anordnungen, die nur eine Lage von parallelen Zählrohren benutzten. Dies bedeutet einen grundsätzlichen Vorteil dieses Prinzips der gekreuzten Zählrohre, das in einfacherer Form schon von $\mathrm{Cl}$ ay und Mitarbeitern angewandt wurde.

Schauer mit größerem Öffnungswinkel werden in entsprechender Weise erfaßt, indem man je drei wei- ter voneinander entfernte Zählrohre zu einer Gruppe zusammenfaßt, so daß die einzelnen Gruppen sich überlappen. Dies wird durch einfaches Umschalten erreicht. Die im folgenden benutzten Gruppierungen sind in Tab. 1 in leicht verständlicher Weise zusammengefaßt. Die Numerierung der Zählrohre ist wie in Abb. 1; von je vier Gruppen einer Zählrohrlage ist nur die erste angegeben. Tab. 1 enthält weiter die entsprechenden mittleren Divergenzwinkel. Die Gesamtbreite der Winkelverteilung beträgt in allen Fällen rd. $7^{\circ}$. Jedoch hat die geometrische Treffwahrscheinlichkeit für Zweistrahlschauer als Funktion des Schauerwinkels eine scharfe Spitze nahe dem mittleren, durch die Quadratlagen bestimmten Winkel. Die Gesamtzahl der Quadratkombinationen ist für alle fünf Winkel dieselbe, nämlich 128. Ebenso sind die totale wirksame Fläche $F$ für den einen der beiden Schauerstrahlen und der mittlere wirksame Raumwinkel $\Omega$ für den anderen Strahl für alle Gruppierungen dieselben. Daher sind die Messungen für die verschiedenen Divergenzwinkel unmittelbar vergleichbar. Man findet

$$
\begin{gathered}
F=12^{2} \cdot 4^{2}=2304 \mathrm{~cm}^{2}, \\
\Omega=\frac{16}{9} \frac{4^{2}}{100 \cdot 93}=3,06 \cdot 10^{-3} .
\end{gathered}
$$

Der Faktor 16/9 in $\Omega$ ist die mittlere Zahl der Quadrate, mit denen ein Quadrat kombinieren kann.

Für die Untersuchung der schauerauslösenden Strahlung wurde noch eine weitere Zählrohrlage III über oder im Innern des Schauerstrahlers angebracht. Diese konnte in verschiedener Weise in Koinzidenz oder Antikoinzidenz zu den Lagen I + II geschaltet werden; hierüber s. Ziff. 7. Schließlich wurden gelegentlich noch Antikoinzidenzzählrohre IV (Abb. 1) angebracht, um seitlich in den Schauerstrahler eintretende ionisierende Teilchen unwirksam zu machen (Ziff. 5).

Die Apparatur war erst im Kellergeschoß (rd. 110 m ü. M.), dann im Dachgeschoß des Universitätsinstitutes aufgestellt, beide Male so, daß der Schauerstrahler sich $1 \mathrm{~m}$ unter der untersten Decke befand. Im Kellergeschoß betrug die Gesamtdicke der darüberliegenden Decken $290 \mathrm{~g} / \mathrm{cm}^{2}$, im Dachgeschoß nur $30 \mathrm{~g} / \mathrm{cm}^{2}$. 


\section{Die Verstärkerschaltungen}

Abb. 2 zeigt das Blockschema der Schaltung. Von dem unteren Teil des Schemas mit der Zählrohrlage III sei zunächst abgesehen. Die positive Zählspannung lag an den Zählrohrdrähten. Die Zählrohrimpulse wurden zunächst durch einen Multivibrator nach dem von $\mathrm{M}$ a i e r-Leibnitz ${ }^{28}$ angegebenen Prinzip in negative Rechteckimpulse von konstanter Höhe und Breite umgeformt. Zur Aussonderung der Vierfach-

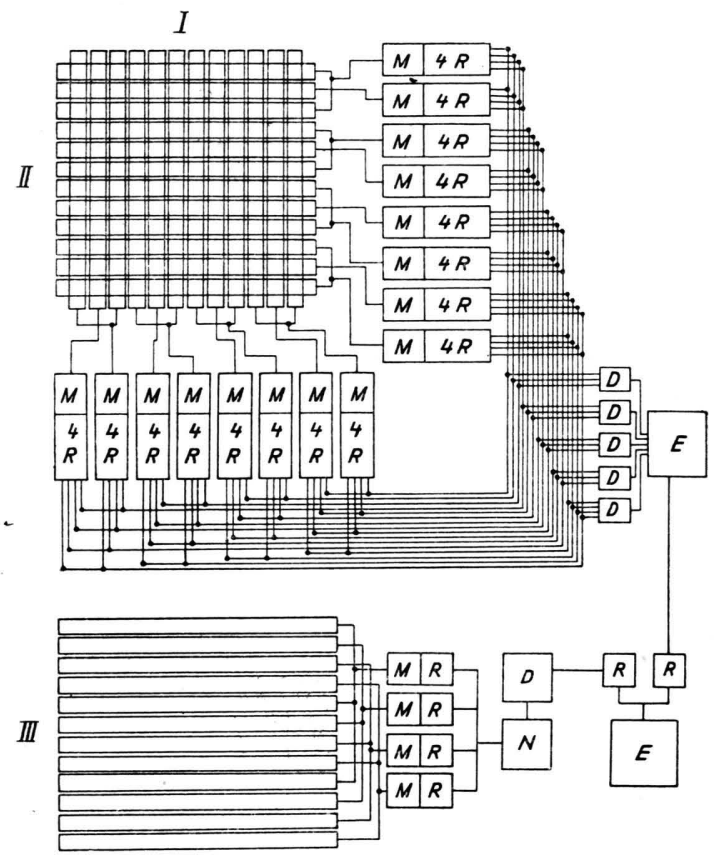

Abb. 2. Blockschema der Verstärkerschaltungen. $M=$ Multivibrator, $\mathrm{R}=$ Rossi-Röhre, $\mathrm{D}=$ Diskriminator, $\mathrm{N}=\mathrm{Nach}^{-} \cdot$ verstärker, $\mathrm{E}=$ Endmultivibrator mit Zählwerk.

Koinzidenzen wurde die von $\mathrm{R}$ os si angegebene Schaltung benutzt. Die Rechteckimpulse jedes Eingangs-Multivibrators wurden einer von vier „RossiRöhren“ zugeführt, die gemeinsamen Anodenwiderstand hatten. Nur wenn alle vier Rossi-Röhren gleichzeitig gesperrt wurden, erfolgte am Ausgang ein kräftiger Impuls. Um alle gewünschten Koinzidenzmöglichkeiten zu erhalten, wurde folgendermaßen verfahren. Jeder der 16 Eingangs-Multivibratoren war anodenseitig an die Gitter von vier Rossi-Röhren angekoppelt, von denen jedoch nur eine einen Anodenwiderstand hatte, die übrigen drei hatten ihre Anodenwiderstände in je einem der damit in Vierfach-Koinzidenz geschalteten Verstärker.

28 H. M a i e r - L e ib n itz, Rev. Sci. Instruments 19, 500 [1948].
Eine Gruppe von vier Rossi-Röhren liefert unvermeidlich auch schon bei einer Dreifach-Koinzidenz Impulse, die zwar viel schwächer sind als die Vierfach-Impulse, aber doch ausgeschieden werden müssen. Hierzu dienen Diskriminatorstufen. Es erwies sich als zweckmäßig, nicht alle 16 Koinzidenzimpulse über einen und denselben Diskriminator zu leiten, sondern sie auf 5 Diskriminatorröhren zu verteilen, damit die Schaltung sicher arbeitet und nicht ein Teil der Koinzidenzimpulse unterschlagen wird. Die aus den Diskriminatoren kommenden Impulse wurden einem gemeinsamen Endmultivibrator zugeführt, der über eine weitere Pentode ein Zählwerk betätigte.

Zur Kontrolle dieser komplizierten Anordnung wurde ein Prüfsystem ausgearbeitet, das ein Versagen eines Verstärkerteiles oder eines Zählrohres sofort erkennen ließ. Die Zählrohre wurden übrigens regelmäßig nach höchstens $10^{8}$ Zählstößen (bei 1600/min) durch neue ersetzt. Die ganze Anordnung erwies sich so als sehr betriebssicher, obwohl sie rd. 110, später bei Mitbenutzung der Zählrohre III und IV sogar 150 Röhren enthielt.

Das Auflösungsvermögen der Koinzidenzschaltung wurde in bekannter Weise aus den Zufallskoinzidenzen zwischen zwei weit entfernten Zählrohren bestimmt. Die Auflösezeit war hauptsächlich durch die Zeitkonstante der Rossi-Stufe bestimmt. Betrug diese $2,3 \cdot 10^{-6} \mathrm{sec}$, so war die Auflösezeit $2,0^{\cdot} \cdot 10^{-6} \mathrm{sec}$, und dies erwies sich gerade als lang genug, daß keine wirklichen Koinzidenzen der Messung entgingen. Zur Sicherheit wurde mit einer Auflösezeit von $5 \cdot 10^{-6} \mathrm{sec}$ gearbeitet. Diese Zeit war immer noch kurz genug, so daß bei den Hauptmessungen die Zufallskoinzidenzen aller Art vernachlässigt werden konnten.

\section{Vorversuche}

Die ersten Versuche galten der Frage, ob das 2. Maximum überhaupt existiert. Bei den Versuchen von $\mathrm{Schmeiser}$ und B othe ${ }^{3}$ war der Schauerstrahler nicht quaderförmig, sondern nach oben hin stufenförmig verbreitert. Es ist behauptet worden ${ }^{23}$, daß durch diese Stufe das 2. Maximum vorgetäuscht wurde, obwohl dieser Einwand schon von Schmeiser und Bothe selbst widerlegt worden ist. Bei allen hier beschriebenen Versuchen wurde der Schauerstrahler stufenfrei mit senkrechten Seitenwänden aufgeschichtet. Schon die ersten kurzen Meßreihen zeigten einwandfrei das 2. Maximum in der erwarteten Höhe.

Von anderer Seite ist vermutet worden, daß das 2. Maximum einen Störeffekt darsteillt, der durch das Holzgestell der Anordnung in irgendeiner Weise ver- 
ursacht wird. Dies wurde in der Weise geprüft, daß von den Zählrohren in I und II nur eine „Sechsergruppe “ (3 unten, 3 oben) angeschaltet wurde. Dann hätte eine Gruppe neben einem der Holzpfosten das 2. Maximum deutlicher zeigen müssen als eine Gruppe in der Mitte. Das Umgekehrte war der Fall. Die Rand- und Eckgruppen ergaben sogar ein etwas flacheres 2. Maximum; dies mag seinen Grund teils darin haben, daß die vertikale Schauerrichtung bevorzugt ist, teils darin, daß infolge des teilweise schrägen Strahlenganges im Schauerstrahler das Maximum verschmiert wird. Wurden neben die angeschalteten Gruppen beiderseits dicke Holzbalken gelegt, so erhöhte sich nur der Untergrund um einen konstanten Betrag (durch Kaskadenschauer aus den Balken), die Form der Auslösekurve und die Höhe des 2. Maximums blieben ungeändert.

Hiermit dürfte erneut sichergestellt sein, daß das 2. Maximum durch Schauer aus dem Bleistrahler hervorgerufen wird. Erst recht lassen die jetzt zu beschreibenden Hauptversuche keinen Zweifel hierüber.

\section{Die Auslösekurve bei verschiedenen Schauerwinkeln im Keller ${ }^{29}$}

Mit allen 24 Zählrohren in den in Tab. 1 angegebenen Gruppierungen wurden im Kellergeschoß die 5 Kurven der Abb. 3 a erhalten (die entsprechenden Kurven für das Dachgeschoß, Abb. $3 \mathrm{~b}$, werden in Ziff. 9 und 10 behandelt). Die zugehörigen mittleren Schauerwinkel sind angeschrieben. Es treten 3 deutliche Maxima auf.

Das 1. Maximum rührt von den Elektronenschauern her, die über dem Meeresniveau noch überwiegend zur weichen Komponente der kosmischen Strahlung gehören. Infolge der Vorfilterung durch die Institutsdecken ist daher das 1. Maximum im Keller relativ niedrig.

Das 2. Maximum liegt hier durchgängig bei $15 \mathrm{~cm}$ $\mathrm{Pb}$, während $\mathrm{S}$ chmeiser und $\mathrm{Bothe} \mathrm{e}^{3}$ es bei rd. $17 \mathrm{~cm} \mathrm{~Pb}$ fanden. Der Unterschied beruht nicht ganz auf Meßfehlern, er rührt im wesentlichen daher, daß bei unseren Messungen schon die Zimmerdecke zum Teil als Schauerstrahler mitwirkte. Schaltet man die Deckenschauer aus, so rückt das 2. Maximum um rd. $1 \mathrm{~cm} \mathrm{~Pb}$ nach rechts (vgl. Ziff. 7). Die Lage des 2. Max. erwies sich zeitlich als völlig konstant, insbesondere unabhängig von meteorologischen Faktoren. Für

29 Über die Ergebnisse von Ziff. 5-8 wurde schon kurz berichtet ${ }^{30}$.

30 W. Bothe u. H. Thurn, Physic. Rev. 79, 544 [1950].

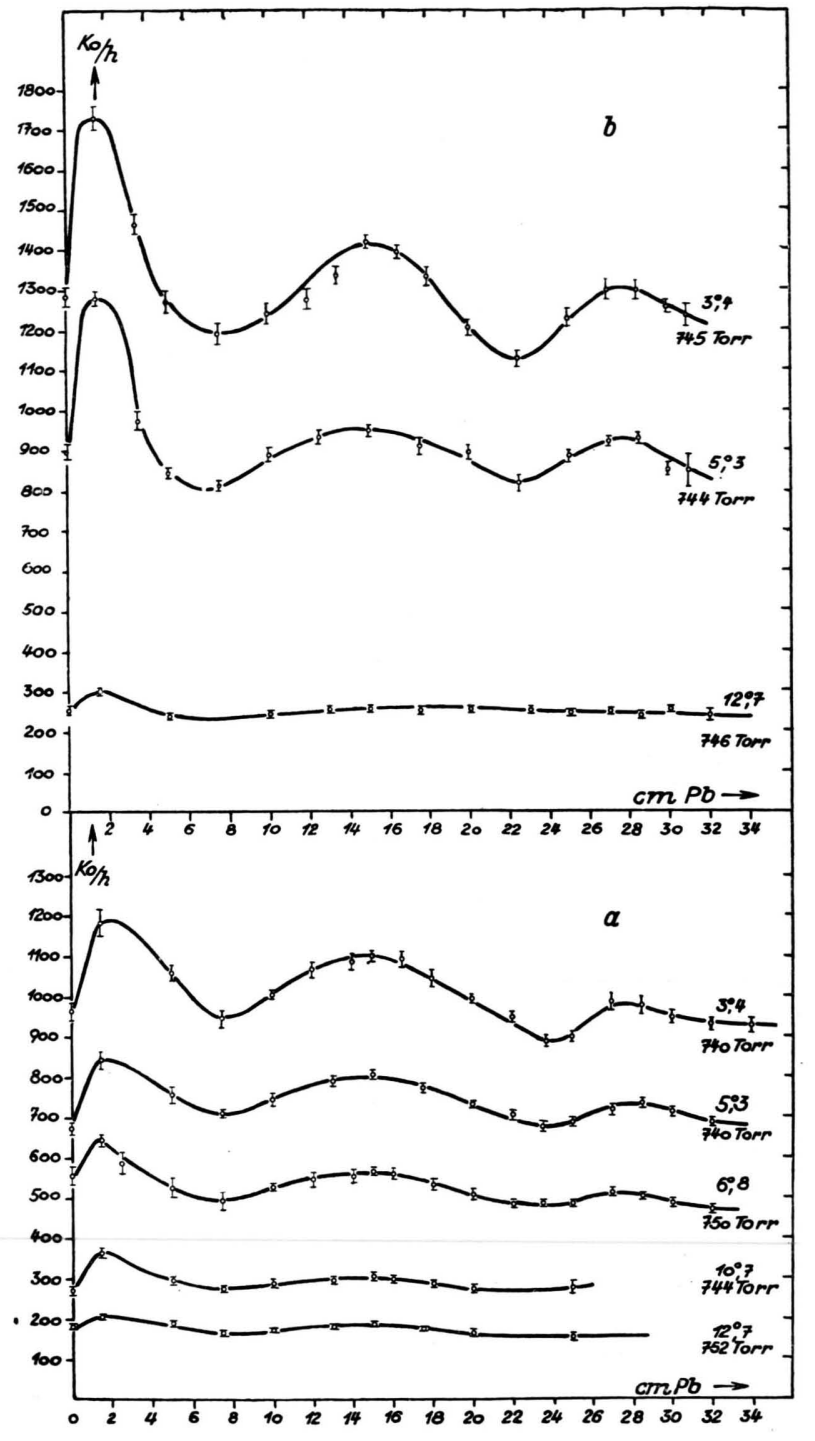

Abb. 3. Auslösekurven bei verschiedenen Schauerwinkeln, a im Kellergeschoß, b im Dachgeschoß.

den Schauerwinkel 3,6 beträgt die Höhe des 2. Max. über dem vorhergehenden Minimum gerade das 16-fache derjenigen, die mit einer einzigen Sechsergruppe (Ziff. 4) beobachtet wurde. Dies zeigt schon, $\mathrm{da} ß$ die 16 Sechsergruppen unabhängig arbeiten, $\mathrm{d}$. h. $\mathrm{da} ß$ die Schauer so eng und/oder so strahlenarm sind, $\mathrm{da} ß$ sie jeweils nur eine Sechsergruppe betätigen; andernfalls müßte das 2. Maximum jetzt niedriger sein. Mit wachsendem Schauerwinkel nimmt nach Abb. 3 die Höhe des 2. Maximums wesentlich rascher ab als die des 1. Maximums, die Schauer sind also wesentlich enger als die Elektronenschauer, wie ebenfalls schon früher ${ }^{3}$ festgestellt. 
Das 3. Maximum ist meist niedriger als das 2. und nimmt mit wachsendem Winkel eher noch rascher $a b$ als dieses. Auch hier handelt es sich also um „enge Schauer". Die Lage des 3. Maximums hängt, im Gegensatz zum 2. Maximum, stark vom Barometerstand $\mathrm{ab}$, wie schon $\mathrm{Abb} 3$ erkennen läßt. Dieser Barometereffekt wird in Ziff. 8 noch ausführlicher behandelt.

Einige Messungen wurden noch in der Weise gemacht, daß an den Seitenflächen des Schauerstrahlers die Antikoinzidenzzähler IV angebracht wurden (Abb. 1). Dies änderte praktisch nichts an dem Kurvenverlauf, nur der Untergrund wurde schwächer. Dies zeigt, daß die in die Seitenflächen eintretenden ionisierenden Strahlen nicht zur Erklärung des 2. und 3. Maximums herangezogen werden können.

\section{Winkelverteilung und Häufigkeit der engen Schauer im Keller}

Der ziemlich hohe Nulleffekt und der allgemeine Untergrund rühren teils von Seitenschauern her, die durch den Schauerstrahler nicht beeinflußt werden, teils von Deckenschauern, die durch den Schauerstrahler in schwer zu übersehender Weise verändert werden. Daher ist eine exakte Zerlegung der Kurven Abb. 3 in ihre $4-5$ Komponenten nicht möglich. Um wenigstens ein grobes $\mathrm{Ma}$ für die Häufigkeit der engen Schauer zu gewinnen, wurde für jeden Winkel die Höhe des 2. und 3. Max. über dem vorausgehenden Minimum abgelesen. Diese Höhen, in Koinz./h, sind in Abb. 4 a und c aufgetragen; sie stellen offenbar nur eine untere Grenze für die Häufigkeit der tatsächlich beobachteten Schauer dar. Die Winkelabhängigkeit kann, wie man sieht, in grober Näherung durch Exponentialfunktionen dargestellt werden. Die Bedeutung dieser Funktionen ist offenbar folgende. Ist $\vartheta$ der Öffnungswinkel, $\varphi$ das Azimut eines Zweistrahlschauers, $N(\vartheta) \mathrm{d} \Omega=N(\vartheta) \mathrm{d} \cos \vartheta \cdot \mathrm{d} \varphi$ die relative Häufigkeit der Schauer, bei denen der eine Strahl in das Raumwinkelelement $\mathrm{d} \Omega$ relativ zum anderen fällt, so wird $N(\vartheta)$, die „Schauerhäufigkeit pro Raumwinkeleinheit", durch die Kurven a und c der Abb. 4 wiedergegeben.

Diese Kurven stellen aber noch nicht ganz die Winkelverteilung der engen Schauer selbst dar. Wie früher ${ }^{3}$ gezeigt, sind die aus dem Schauerstrahler kommenden engen Schauer im allgemeinen von weicheren Tertiärstrahlen, vermutlich Stoßelektronen, begleitet, die eine breitere Winkelverteilung haben und daher eine zu große Winkeldivergenz vortäuschen.
Diese Tertiärelektronen können praktisch ausgeschaltet werden, indem man die Zählrohre mit einem $1 \mathrm{~cm}$ $\mathrm{Pb}$-Absorber bedeckt ${ }^{31}$. Solche Messungen wurden für die beiden WinkeI $\vartheta=5^{\circ} 3$ und 12,7 durchgeführt (Abb. 5). Bei so großen Zählerabständen können auch Stoßelektronen aus dem Absorber keine zu-

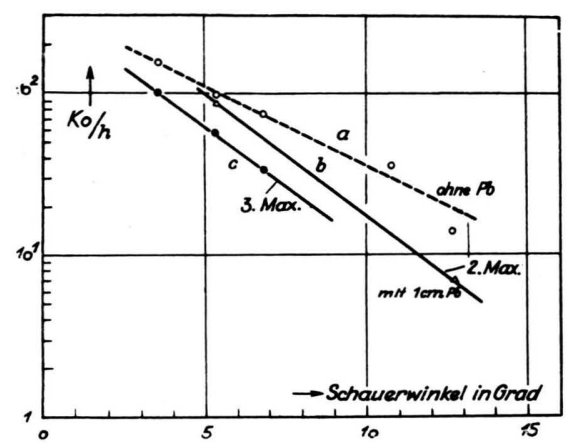

Abb. 4. Winkelverteilung der Schauer des 2. und 3. Max.

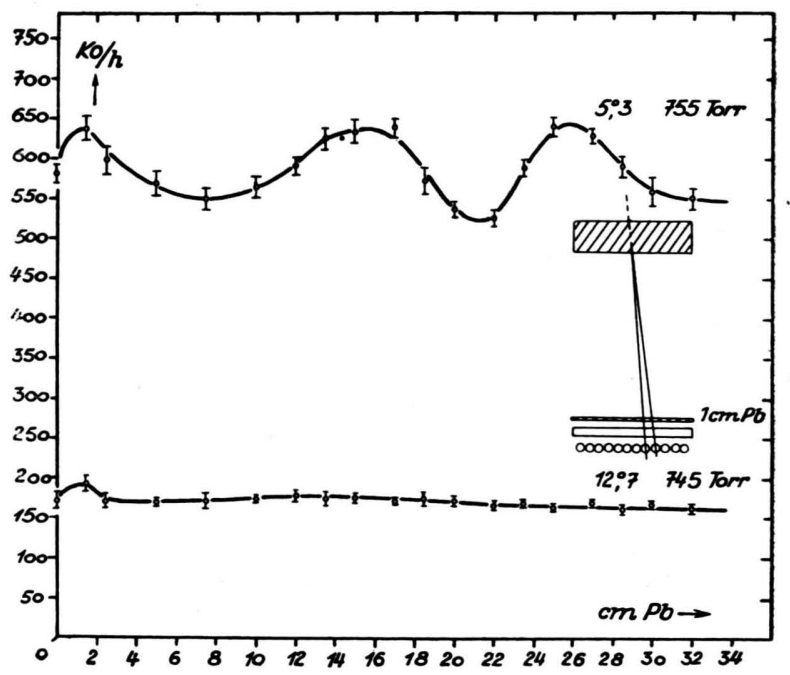

Abb. 5. Auslösekurven mit $1 \mathrm{~cm}$ Bleifilter über den unteren Zählrohren.

sätzlichen Koinzidenzen hervorrufen ${ }^{32}$. Bei 5,3 war das 1. Maximum wesentlich niedriger (Absorption der Elektronenschauer), das 2. Maximum etwa ebenso hoch und das 3. Maximum höher als in der entsprechenden Kurve von Abb. 4; letzteres braucht nicht mit dem Absorber zusammenzuhängen, da die Höhe des 3. Maximums auch sonst anscheinend unsystematische

31 W. E. H a z e n, Physic. Rev. 64, 7 [1943].

32 W. W. B r own, A. S. M c K a y u. E. D. P a l m a ti e r, Physic. Rev. 76, 506 [1949]. 
Schwankungen zeigte. In der Kurve für $12^{\circ}, 7$ war das 2. Maximum fast ganz, das 3. vollständig verschwunden. Die wahre Verteilung der Schauerwinkel wird daher etwa durch die ausgezogenen Geraden b und c der Abb. 4 wiedergegeben. Diese ergeben

$$
\begin{aligned}
N(\vartheta) & =N_{0} e^{-\vartheta / \vartheta_{0}} ; \quad \vartheta_{0} \approx 3^{0} ; \\
N_{0} & =500 / \text { h für das 2. Max. } \\
N_{0} & =350 / \text { h für das 3. Max. }
\end{aligned}
$$

Es sei nun $n(\vartheta) \mathrm{d} \vartheta$ die gesuchte Zahl der Zweistrahlschauer pro $\mathrm{cm}^{2}$ und Stunde, deren Öffnungswinkel zwischen $\vartheta$ und $\vartheta+\mathrm{d} \vartheta$ liegt. Dann ist die beobachtete Zahl pro Stunde offenbar

$$
N(\vartheta)=n(\vartheta) F \Omega /(2 \pi \vartheta),
$$

wo $F=2304 \mathrm{~cm}^{2}$ die gesamte gemeinsame Zählfläche der Lagen I und II und $\Omega=3,06 \cdot 10^{-3}$ der gesamte mittlere Raumwinkel ist, der dem einen Schauerstrahl zur Verfügung steht, wenn der andere ein bestimmtes empfindliches Quadrat trifft (Ziff. 2). Damit wird

$$
n(\vartheta)=\frac{2 \pi \vartheta N(\vartheta)}{F \Omega}=\frac{2 \pi N_{0}}{F \Omega} \text { if } e^{-\vartheta / \vartheta_{0}}
$$

und die Gesamthäufigkeit

$$
n_{\mathrm{s}}=\int n \mathrm{~d} \vartheta=\frac{2 \pi N_{0} \vartheta_{0}{ }^{2}}{F \Omega} .
$$

Mit den obigen experimentellen Werten von $N_{0}$ und $\vartheta_{0}$ wird für die Schauer des 2. Max. $n_{\mathrm{s}}=1,2 / \mathrm{cm}^{2} \cdot \mathrm{h}$. Statt der Exponentialfunktion kann man auch fast ebenso gut eine Gauß-Funktion an die Beobachtungen anpassen. Die entsprechende Rechnung ergibt dann praktisch denselben Wert $n_{\mathrm{s}}$.

Dennoch ist diese Berechnung recht unsicher, u. a. deshalb, weil dabei vorausgesetzt ist: $\Omega \ll \vartheta^{2}$, was nicht annähernd zutrifft. Deshalb wurde noch eine unabhängige Bestimmung von $n_{\mathrm{s}}$ durch einen „Monte Carlo“-Versuch vorgenommen: eine große Zahl von Metalldrähten, deren Längen nach dem Gesetz (1) verteilt waren, wurde wiederholt ungeordnet auf eine Nachzeichnung der gekreuzten Zählrohrlagen geworfen und die Zahl der „Koinzidenzen“ zwischen den Drahtenden festgestellt, und zwar für die verschiedenen Zählrohranordnungen der Tab. 1. Man erhielt die Kurve b der Abb. 4 mit den richtigen Ordinaten, wenn insgesamt 4000 Drähte geworfen wurden, d.h. 4000 Schauer/h trafer, die Zählrohrlage, oder $n_{\mathrm{s}}=1,4 / \mathrm{cm}^{2} \cdot \mathrm{h}$. Diese $n_{\mathrm{s}}$ sind, wie oben bemerkt, Minimalwerte. Man kann rechnen mit rd. 2 Schauern $/ \mathrm{cm}^{2} \cdot \mathrm{h}$ im 2. Maximum im Kellergeschoß. Die Schauer des 3. Maximums sind durchschnittlich etwas weniger häufig.

Diese Berechnung setzt voraus, daß 1. die Schauer nicht mehr als zwei Strahlen enthalten, und daß 2. die Zählrohre auf jeden durchgehenden Schauerstrahl ansprechen. Andernfalls kann das Ergebnis leicht nachträglich umgedeutet werden. Die beiden erwähnten Punkte werden w. u. noch eingehender geprüft (Ziff. 12 und 13).

7. Die schauerauslösenden Strahlungen

Um etwas über die Natur der einfallenden Strahlungen zu erfahren, die im Blei die Schauer des 1., 2. und 3. Maximums hervorrufen, wurde die Anordnung ergänzt durch die Zählrohrlage III über dem Schauerstrahler (Abb. 1). Diese bestand ebenfalls aus 12 Zählrohren derselben Art und Größe wie in den Lagen I und II. Von diesen wurden je drei in einem Abstande von je $18 \mathrm{~cm}$ parallelgeschaltet und an einen Multivibrator gekoppelt, wie im unteren Teil des Blockschemas Abb. 2 dargestellt. Diese 4 Dreiergruppen konnten nun durch entsprechende Einstellung der Diskriminatoren in verschiedener Weise mit den Vierfach-Koinzidenzen der Lagen I + II in Koinzidenz, und außerdem auch in Antikoinzidenz geschaltet werden. Dabei wurden die Zeitkonstanten der Zählrohre III etwas größer als bei I + II gewählt, damit möglichst keine Koinzidenzen oder Antikoinzidenzen verlorengingen. Es wurden folgende drei Schaltungen benutzt:

a) Angezeigt wurden nur Fünffach-Koinzidenzen, die durch Koinzidenz einer und nur einer Dreiergruppe in III mit I + II entstanden. Bei den großen Zählrohrabständen innerhalb einer Dreiergruppe bedeutet das praktisch, daß die beobachteten Schauer durch einen ionisierenden Einzelstrahl ausgelöst wurden. Mit dieser Schaltung wurden die Kurven $a_{1}$ und $a_{2}$ der Abb. 6 erhalten.

b) Angezeigt wurden Siebenfach-Koinzidenzen, die durch Koinzidenz von mindestens dreien der vier Dreiergruppen in III mit I + II entstanden. So erhält man die Schauer, die durch einfallende Schauer von mindestens drei ionisierenden Teilchen ausgelöst werden. Das Ergebnis für den Winkel $3{ }^{\circ} 6$ zeigt Kurve b der Abb. 6.

c) Alle Zählrohre III wurden in Antikoinzidenz zu I + II geschaltet; hierzu war noch eine Umkehrstufe in den Ausgang von III eingeschaltet. Auf diese Weise wurden, abgesehen von den Seitenschauern, nur solche Schauer erfaßt, die im Blei durch nichtionisierende 
Strahlen ausgelöst wurden. Das Ergebnis für den Schauerwinkel 3,6 ist in Kurve c der Abb. 6 wiedergegeben.

Wie Abb. 6 zeigt, ist es durch diese Versuche gelungen, jedes der 3 Maxima von den beiden anderen $\mathrm{zu}$ isolieren. Das 1. Maximum tritt nur in der Kurve b auf. Dies ist so zu deuten, daß die im Blei entstehenden Elektronenkaskaden nur Weiterentwicklungen der von oben auffallenden Kaskaden sind. Diese entstehen in leichtatomigen Materialien und dürften nach Durchlaufen der Betondecken schon fast abgestorben

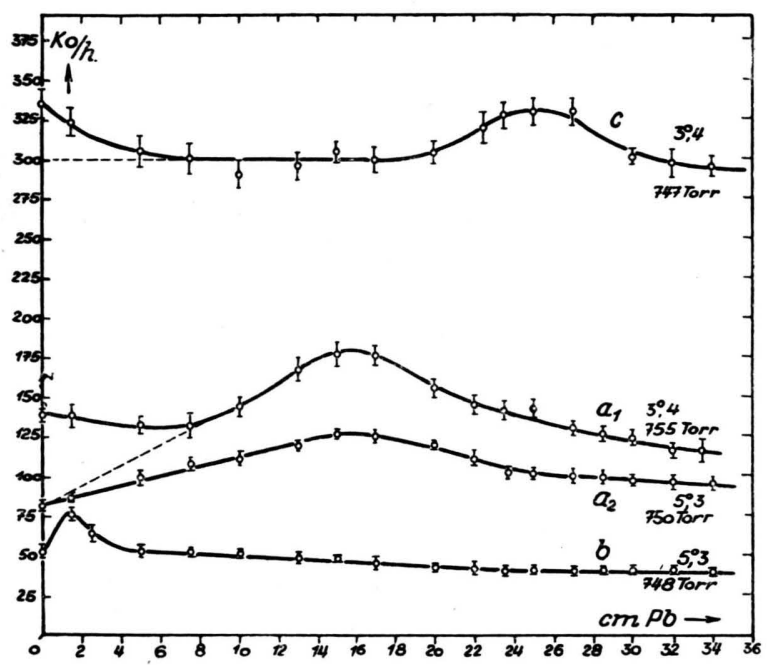

Abb.6. Auslösekurven für verschiedene Primärstrahlungen: $a_{1}, a_{2}$ für geladene Einzelstrahlen; $b$ für einfallende Schauer; c für neutrale Primärstrahlung.

sein, sie können sich aber im Blei erneut multiplizieren, weil die „kritische Energie“ im Blei wesentlich tiefer liegt als im Beton ${ }^{33}$.

Das 2. Maximum findet sich nur in den beiden a-Kurven der Abb.6. Hier kann das 2. Maximum nur durch ionisierende Einzelteilchen verursacht sein. Nichtionisierende Strahlen und teilchenreiche Schauer kommen als Auslösende nicht in Frage, wie die Kurven $b$ und $c$ zeigen ${ }^{34}$. In den a-Kurven ist das 2 . Maximum deutlich flacher als in den entsprechenden Kurven der Abb. 3. Es ist schwer, mit Sicherheit zu

33 B. R os s i u. K. Gre is e n, Rev. mod. Physics 13, 240 [1941].

34 In der Lage der Meßpunkte um $16 \mathrm{~cm} \mathrm{~Pb}$ bei Kurve c könnte man vielleicht noch eine schwache Andeutung des 2. Maximums erkennen, was sich aber ohne weiteres daraus erklären würde, daß durch die engen Zwischenräume zwischen den Zählrohren III doch noch einzelne Ionisierende dringen konnten, ohne Antikoinzidenzen hervorzurufen. entscheiden, ob dieser Unterschied apparative Ursachen hat. Andernfalls wäre zu schließen, daß der auslösende Strahl noch von einem zweiten ionisierenden Strahl begleitet sein kann. Auch nichtionisierende Begleitstrahlen sind natürlich nicht ausgeschlossen.

Die Kurve $a_{1}$ enthält offenbar noch eine Komponente, die mit zunehmender Dicke des Schauerstrahlers monoton abnimmt und bei $\mathrm{rd} .8 \mathrm{~cm} \mathrm{~Pb}$ praktisch verschwunden ist. Dies erklärt sich zwanglos daraus, daß schon von der Zimmerdecke enge Schauer kommen, die dann in dem eigentlichen Schauerstrahler absorbiert werden. Im Falle der Kurve $a_{1}$ konnten sehr enge Deckenschauer zur Wirkung kommen, deren beide Partner durch ein und dasselbe Zählrohr III gingen und deshalb angezeigt wurden. Bei dem größeren Winkel der Kurve $a_{2}$ war dies nicht mehr möglich, es mußten zwei Zählrohre III durchlaufen werden, so daß hier die Deckenschauer ausgeschieden wurden.

Die Deckenschauer liefern auch die Erklärung dafür, daß in den a-Kurven das 2 . Maximum bei $16 \mathrm{~cm}$, in Abb. 3 dagegen bei $15 \mathrm{~cm} \mathrm{~Pb}$ liegt. Ohne die Zählrohre III werden einige Deckenschauer des 2. Maximums mitgemessen. Die Decke wirkt zum Teil schon als Schauerstrahler mit, das 2. Maximum rückt daher scheinbar zu kleineren Dicken. Die wirkliche Lage des 2. Maximums ist demnach bei $16 \mathrm{~cm} \mathrm{~Pb}$.

Das 3. Maximum schließlich tritt nur in der Kurve c der Abb. 6 auf, kann also nur durch nichtionisierende Auslösende entstanden sein. Es ist deutlich flacher als in Abb. 3; hierfür kann eine sichere Deutung vorerst nicht gegeben werden. Der schwache Abfall am Anfang der Kurve c erklärt sich zwanglos durch Deckenund Seitenschauer.

\section{Der Barometereffekt im 3. Maximum}

Während das 2. Maximum immer an derselben Stelle gefunden wurde, schwankte die Lage des 3. Maximums in ziemlich weiten Grenzen. Tab. 2 ist eine Zusammenstellung aller Messungen, aus denen die Lage des 3. Maximums mit ausreichender Genauigkeit entnommen werden konnte. Die Versuchsbedingungen und einige atmosphärische Daten sind angegeben; die letzteren wurden dem „Täglichen Wetterbericht" des deutschen Wetterdienstes für Heidelberg entnommen. Die hiernach gezeichnete Abb. 7 zeigt, daß ein streng gesetzmäßiger Zusammenhang besteht zwischen dem auf Meeresniveau reduzierten Barometerstand $B[\mathrm{~cm} \mathrm{Hg}]$ und der Lage 


\begin{tabular}{|c|c|c|c|c|c|c|c|c|c|c|c|c|}
\hline A & B & $\mathrm{C}$ & D & $\mathrm{E}$ & $\mathrm{F}$ & G & \multicolumn{6}{|c|}{$\mathrm{H}$} \\
\hline & \multirow{2}{*}{ Art der Messung } & \multirow{2}{*}{$\begin{array}{l}\overline{0} \\
\overline{3} \\
3\end{array}$} & \multirow{2}{*}{ 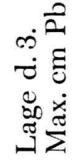 } & \multirow{2}{*}{ 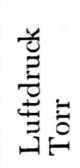 } & \multirow{2}{*}{ 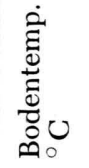 } & \multirow{2}{*}{ 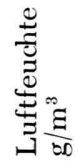 } & & \multicolumn{2}{|c|}{225 mb-Fläche } & \multicolumn{2}{|c|}{96 mb-Fläche } \\
\hline & & & & & & & $\begin{array}{l}\text { Höhe } \\
m\end{array}$ & $\underset{{ }^{\circ} \mathrm{C}}{\mathrm{Temp}}$ & $\begin{array}{l}\text { Höhe } \\
m\end{array}$ & $\underset{{ }^{\circ} \mathrm{C}}{\mathrm{Temp}}$ & $\underset{m}{\text { Höhe }}$ & $\underset{{ }^{\circ} \mathrm{C}}{\mathrm{Temp}}$ \\
\hline 1 & $\begin{array}{l}\text { Antiko. Seitenzählr. z. } \\
\text { 4-f.-Ko. I + II (Keller) }\end{array}$ & 3,4 & 29,0 & 735 & +4 & 6,40 & 5320 & -30 & 10800 & 0 & 16300 & +10 \\
\hline 2 & $\begin{array}{l}\text { 4-fach-Koinz. I + II } \\
\text { (Keller) }\end{array}$ & 3,4 & 28,0 & 740 & +13 & 7,05 & 5490 & -20 & 10840 & -22 & 16390 & -44 \\
\hline 3 & $\begin{array}{l}\text { 4-fach-Koinz. I + II } \\
\text { (Keller) }\end{array}$ & 5,3 & 28,5 & 740 & +8 & 5,10 & 5290 & -28 & 10660 & -42 & 16320 & -42 \\
\hline 4 & $\begin{array}{r}\text { 4-fach-Ko. I + II } \\
\text { (Dachgeschoß) }\end{array}$ & 5,3 & 28,0 & 744 & -2 & 6,00 & 5365 & -25 & 10715 & +4 & 16230 & +8 \\
\hline 5 & $\begin{array}{r}\text { 4-fach-Ko. I + II } \\
\text { (Dachgeschoß) }\end{array}$ & 3,4 & 27,6 & 745 & +1 & 6,40 & 5300 & -37 & 10530 & +3 & 16220 & +8 \\
\hline 6 & $\begin{array}{l}\text { 4-fach-Koinz. I + II } \\
\text { (Keller) }\end{array}$ & 6,8 & 27,0 & 750 & +17 & 7,80 & 5590 & -21 & 10980 & +3 & 16460 & -48 \\
\hline 7 & $\begin{array}{l}\text { 4-f.-Ko. I + II Kontrolle } \\
\text { (Keller) }\end{array}$ & 3,4 & 27,0 & 750 & +11 & 7,30 & 5710 & -22 & 11230 & +8 & 16470 & +8 \\
\hline 8 & $\begin{array}{l}\text { 4-f.-Ko. I + II mit } \\
1 \text { cm Pb-Abs. (Keller) }\end{array}$ & 5,3 & 25,8 & 755 & +21 & 8,45 & 5690 & -13 & 11220 & +3 & 16690 & +3 \\
\hline 9 & $\begin{array}{c}\text { Antiko. III zu 4-f.-Ko.I + II } \\
\text { (Keller) }\end{array}$ & 3,4 & 26,0 & 755 & +11 & 4,77 & 5590 & -22 & 10890 & +9 & 16220 & +6 \\
\hline
\end{tabular}

Tab. 2. Lage des 3. Maximums.

des 3. Maximums $M[\mathrm{~cm} \mathrm{~Pb}]^{35}$, nämlich

$$
M=26,8-1,6(B-76),
$$

oder: $1 \mathrm{~g}$ Luft ist 1,34 g Blei „äquivalent“. Auch zu der absoluten Bodenfeuchtigkeit besteht eine gewisse Korrelation, die aber bei weitem nicht so streng ist.

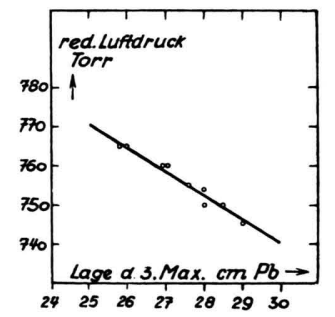

Abb. 7. Abhängigkeit der Lage des 3. Maximums vom reduzierten Luftdruck.

Ein Zusammenhang mit der Bodentemperatur ist nicht erkennbar.

Trägt man die Lage des 3. Maximums gegen die Höhe oder die Temperatur der Isobarenflächen auf,

35 Qualitative Angaben über einen Barometereffekt im 3. Maximum machen auch $\mathrm{F}$ enyves $\mathrm{u}$. Haim a ${ }^{26}$. so ist schon für $500 \mathrm{mb}$ die Korrelation deutlich schwächer als in Abb. 7, und bei $96 \mathrm{mb}$ verschwindet sie ganz. Es wurde noch versucht, ob man durch Berücksichtigung der jahreszeitlichen Schwankungen in der Höhe der Isobarenflächen zu einer stärkeren Korrelation kommen kann. Für die 96 mb-Fläche erreicht man zwar eine kleine Verbesserung, aber bei weitem nicht einen so eindeutigen Zusammenhang wie mit dem Bodenluftdruck. Hiernach scheint also von allen atmosphärischen Faktoren allein der Bodenluftdruck mit der Lage des 3. Maximums direkt gekoppelt zu sein.

Ob für die Höhe des 2. und 3. Maximums ein Barometereffekt existiert, konnte mit der hier erreichten Meßgenauigkeit nicht entschieden werden.

\section{Einflußder Vorfilterung auf die Lage der Maxima}

Um weitere Einblicke in den Entstehungsmechanismus der engen Schauer zu gewinnen, wurden vergleichende Messungen mit verschiedenen und verschieden angeordneten Materieschichten über der Gesamtanordnung gemacht. 
a) Die Apparatur befand sich im Keller unter Betondecken von insgesamt $290 \mathrm{~g} / \mathrm{cm}^{2}$, wie bei den Messungen von Ziff. 5. Diese Vorfilterung wurde jetzt erhöht, indem auf die unterste Decke, $1 \mathrm{~m}$ über dem Schauerstrahler, eine zusätzliche Schicht von Graphit von $43 \mathrm{~g} / \mathrm{cm}^{2}$ gelegt wurde. Die Schicht war genügend ausgedehnt, um den ganzen Raumwinkelbereich der Apparatur zu bedecken. Diese Schicht bewirkte eine gerade noch erkennbare Verschiebung sowohl des 2. als auch des 3 . Maximums um 0,5 bis $1 \mathrm{~cm} \mathrm{~Pb} \mathrm{zu}$ kleineren Dicken.

Würde die Graphitschicht ebenso wirken wie eine äquivalente Luftmasse, so wäre nach Ziff. 8 eine Verschiebung des 3 . Maximums um $5 \mathrm{~cm} \mathrm{~Pb}$ nach links zu erwarten gewesen. Die beobachtete Verschiebung ist viel kleiner und kann trivial gedeutet werden: Schon die unterste Decke wirkt, wenn auch nicht mit ihrer vollen Stärke, als ein Teil des Schauerstrahlers, wie in Ziff. 7 gezeigt, und drückt daher die Maxima etwas nach links. Dieser Effekt muß natürlich bei Verstärkung der Decke noch stärker auftreten, und zwar sowohl beim 2. als beim 3. Maximum, wie auch beobachtet wurde.

b) Um die Vorfilterung zu verringern, wurde die Apparatur aus dem Keller in das Dachgeschoß verbracht; hier befanden sich nur rd. $30 \mathrm{~g} / \mathrm{cm}^{2}$ leichtes Deckenmaterial $1 \mathrm{~m}$ weit über dem Schauerstrahler. Es wurden die der Abb. 3 a entsprechenden Kurven Abb. $3 \mathrm{~b}$ aufgenommen. Die Maxima liegen an denselben Stellen, wo sie im Kellergeschoß bei gleichem Barometerstand gelegen hätten. Wenn die zwischenliegenden Decken von $260 \mathrm{~g} / \mathrm{cm}^{2}$ ebenso wirken würden wie eine entsprechende Änderung des Luftdrukkes, so müßte das 3. Maximum jetzt weit außerhalb des Meßbereiches liegen.

c) Die Apparatur befand sich wieder im Dachgeschoß. Die Zimmerdecke wurde verstärkt, aber nicht wie bei Versuch a) mit Graphit, sondern mit $5 \mathrm{~cm}$ Blei in genügender Ausdehnung. Außerdem waren jetzt über dem Schauerstrahler die Zählrohre III angebracht und mit I + II in Antikoinzidenz geschaltet, wie bei dem Versuch Ziff. 7 c. Die Anordnung und das Ergebnis der. Messung ist in Abb. 8 a gezeigt. Das 3. Maximum ist gegenüber der dem Barometerstand entsprechenden Normallage um $1,7 \mathrm{~cm} \mathrm{~Pb}$ nach links verschoben. Diese Verschiebung kann nicht wie bei Versuch a) trivial gedeutet werden, weil die Deckenschauer jetzt durch Antikoinzidenzen ausgeschaltet sind. Andererseits sieht man jedoch, daß das $\mathrm{Pb}-\mathrm{Filter}$ nicht mit seiner vollen Dicke, sondern nur mit $1 / 3$ seiner Dicke dem Schauerstrahler zuzurechnen ist. d) Der Versuch c) wurde wiederholt mit dem Unterschied, daß das 5-cm-Pb-Filter jetzt nicht über der Zimmerdecke, sondern unmittelbar über den Antikoinzidenzzählern III angebracht wurde. Anordnung und Ergebnis zeigt Abb. 8 b. Der Barometerstand war praktisch derselbe wie beim Versuch c). Die Verschiebung des 3 . Maximums beträgt jetzt $4,5 \mathrm{~cm} \mathrm{~Pb}$, also fast die volle Filterdicke.

In diesem Zusammenhang ist noch besonders bemerkenswert, daß Pri e b s c ${ }^{8}$ sogar in einer Höhe von $2300 \mathrm{~m}$ ü. M. (lokaler Luftdruck 580 Torr) das 3. Maximum an derselben Stelle beobachtet hat, wo es in Heidelberg gefunden worden wäre.

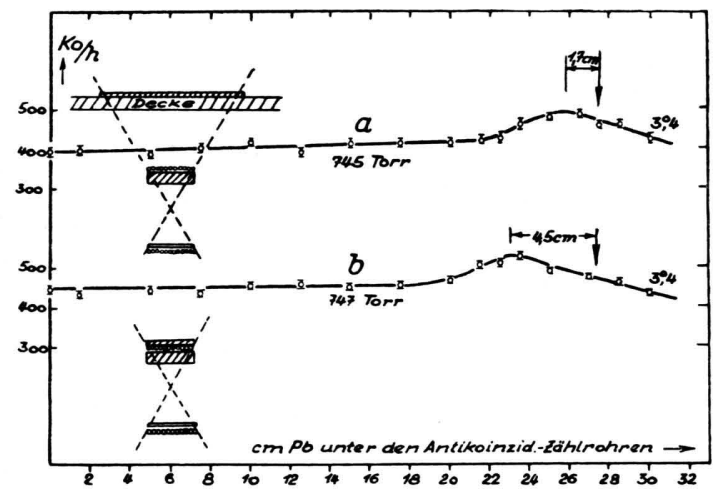

Abb. 8. Auslösekurven für neutrale Primärstrahlen bei verschiedener Anordnung eines 5-cm-Blei-Primärfilters.

Die Gesamtheit dieser Versuche, zusammen mit dem Barometereffekt (Ziff. 8), besagt, daß für die Lage des 3. Maximums nicht allein die Gesamtmasse der über der Apparatur liegenden Materie entscheidend ist, sondern auch ihre vertikale Verteilung. Dies ist ein Hinweis darauf, daß instabile Teilchen von kurzer Lebensdauer an den Vorgängen beteiligt sind.

Der Versuch d) ist noch in anderer Hinsicht interessant. Durch die Zählrohre III werden alle Schauer ausgeschieden, die in dem 5-cm-Pb-Filter entstehen. Trotzdem ist das Ergebnis praktisch dasselbe, wie wenn die Zählrohre III über dem Filter gelegen hätten, d. h. das 3. Maximum liegt bei fast derselben Gesamtdicke $\mathrm{Pb}$. Hieraus kann man schließen, daß in den obersten $5 \mathrm{~cm}$ des Schauerstrahlers fast keine Schauer des 3. Maximums entstehen, daß aber die Qualität der auslösenden nichtionisierenden Strahlung eine Änderung erfährt.

Schließlich ist am Versuch d) noch bemerkenswert, daß wiederum, wie in Ziff. $7 \mathrm{c}$, das 2 . Maximum nicht auftritt, weder an der ursprünglichen noch an einer anderen Stelle. 
10. Einfluß der Vorfilterung auf die Höhe der Maxima; Absorbierbarkeit der schauerauslösenden Strahlungen

Aus den Kurven Abb. 3 ergibt sich, daß im Kellergeschoß das 2. und 3. Maximum im Mittel um rd. 1/3 niedriger ist als im Dachgeschoß. Ein Unterschied im Verhalten der beiden Maxima konnte nicht sicher festgestellt werden. Ebenso war das Verhältnis für die verschiedenen Schauerwinkel praktisch dasselbe, die Winkelverteilung war also oben und unten praktisch dieselbe.

Eine Absorption um 33\% durch $260 \mathrm{~g} / \mathrm{cm}^{2}$ Dekkenmaterial entspricht einer Absorptionslänge von $650 \mathrm{~g} / \mathrm{cm}^{2}$. Dies ist eher eine untere Grenze, weil im Kellergeschoß die auslösenden Strahlen zum großen Teil noch sehr dicke Seitenwände schräg zu durchsetzen hatten. In jedem Falle ist diese Absorptionslänge viel größer als die der weichen Komponente der Ultrastrahlung, die etwa $150 \mathrm{~g} / \mathrm{cm}^{2}$ beträgt ${ }^{36}$. Die Schauer des 2. und 3. Maximums müssen also irgendwie aus der harten (oder einer noch unbekannten) Komponente entstehen, wie früher ${ }^{3}$ schon in bezug auf das 2. Maximum geschlossen wurde.

11. Die Absorbierbarkeit der Schauer

Früher ${ }^{3}$ war nur die Absorptionskurve für die $\mathrm{Ge}$ samtstrahlung im 2. Maximum, also einschließlich des Untergrundes gemessen worden. Jetzt wurden die vollständigen Auslösekurven aufgenommen, nachdem verschieden starke Bleiabsorber A (Abb. 1) über den Zählrohren I + II angebracht waren. Die Absorber begannen $2 \mathrm{~cm}$ über den Zählrohren, als Schauerwinkel wurde 5,3 gewählt. Unter diesen Bedingungen können Stoßelektronen aus dem Absorber praktisch nicht zu den beobachteten Koinzidenzen beitragen $^{32}$. Abb. 9 zeigt die Ergebnisse. Für die Höhe des 2. und 3. Maximums leitet man hieraus etwa dieselbe Absorptionslänge von $9 \mathrm{~cm} \mathrm{~Pb}=100 \mathrm{~g} / \mathrm{cm}^{2} \mathrm{ab}$, für das 1. Maximum dagegen nur rd. $36 \mathrm{~g} / \mathrm{cm}^{2}$, in Übereinstimmung mit früheren Messungen ${ }^{37}$. Die Schauer des 2. und 3. Maximums sind also rd. dreimal so durchdringend wie die Elektronenkaskaden. Könnte man annehmen, daß die beiden Partner eines Schauers gleich durchdringend sind, so müßte jeder eine $\mathrm{Ab}$ sorptionslänge von $200 \mathrm{~g} / \mathrm{cm}^{2}$ haben. Allgemeiner ist zu schließen, daß die Absorptionslänge für beide Partner $>100 \mathrm{~g} / \mathrm{cm}^{2}$, und für einen von ihnen

36 B. Ross i, Rev. mod. Physics 20, 537 [1948].

37 J. J a n o s s y, Cosmic Rays, Oxford 1948, S. 233.
$>200 \mathrm{~g} / \mathrm{cm}^{2}$ sein muß (es addieren sich die $\mathrm{Ab}$ sorptionskoeffizienten der beiden Partner).

Die Gesamtheit dieser Ergebnisse läßt es ziemlich ausgeschlossen erscheinen, daß die engen Schauer aus Elektronen und/oder Photonen bestehen, wie schon früher ${ }^{3}$ geschlossen.

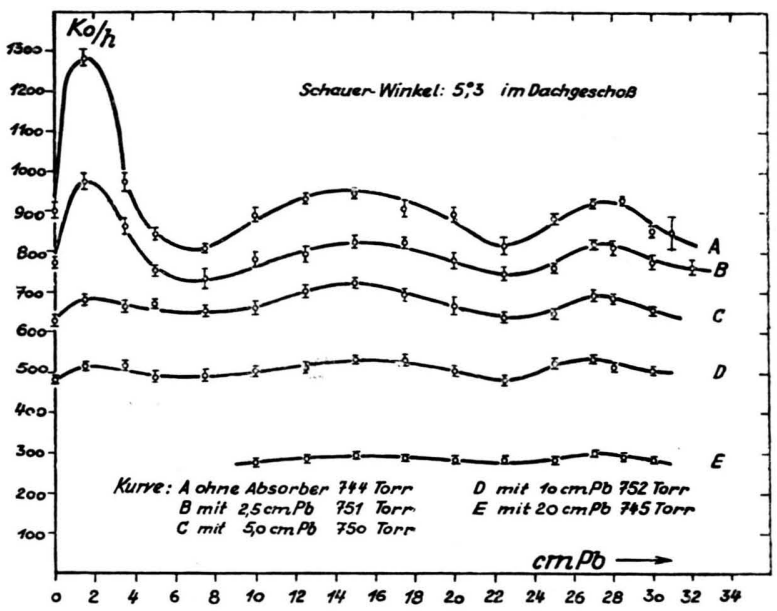

Abb. 9. Auslösekurven mit Bleiabsorbern über den unteren Zählrohren.

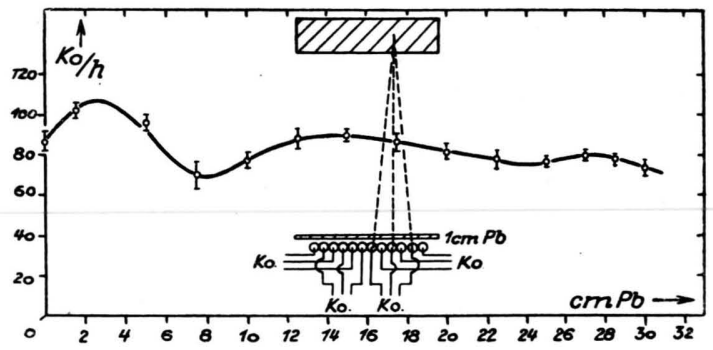

Abb. 10. Auslösekurve mit Dreistrahl-Koinzidenzen.

\section{Mehrstrahlschauer}

Priebs ch ${ }^{8}$ hat das 2. und 3. Maximum beobachtet mit drei in Dreifach-Koinzidenz geschalteten Zählrohren unter dem Schauerstrahler. Clay und Mit$\operatorname{arbeiter}^{15}$ haben dies hinsichtlich des 2. Maximums bestätigt. Wir haben entsprechende Messungen gemacht, jedoch mit dem Unterschied, daß die Zählrohre mit einem $1 \mathrm{~cm}$ starken Bleifilter bedeckt waren, um weiche Tertiärteilchen (Elektronen) auszuschalten, durch die Dreistrahlschauer vorgetäuscht werden könnten. Die Anordnung und das Ergebnis zeigt Abb. 10. 12 parallele Zählrohre wurden zu je dreien mit $9 \mathrm{~cm}$ Abstand in Dreifach-Koinzidenz geschaltet. Das Bleifilter lag $2 \mathrm{~cm}$ über den Zählrohren; Tertiär- 
elektronen aus dem Absorber sollten bei dieser Anordnung nicht stören, weil sie unter nahezu $90^{\circ}$ ausgelöst sein müßten, also nur sehr kleine Energie hätten ${ }^{23}$. In der so gemessenen Auslösekurve (Abb. 10) ist das 2. Maximum noch gut ausgeprägt. Ein Vergleich mit den Zweifach-Koinzidenzen und eine einfache Überschlagsrechnung führen zu dem Ergebnis, $\mathrm{da} ß$ größenordnungsmäßig alle Schauer des 2. Maximums aus drei oder mehr Strahlen bestehen müssen, falls alle Schauerteilchen geladen sind (andernfalls noch mehr). In diesem Punkte sind also die früheren Ergebnisse zu korrigieren.

Das 3. Maximum ist in Abb. 10 nur noch schwach angedeutet. Immerhin ist damit zu rechnen, daß die Schauer des 3. Maximums wenigstens zu einem gewissen Bruchteil aus mehr als zwei Strahlen bestehen.

\section{Naturder Schauerstrahlen}

Bisher liegt noch kein Experiment vor, das Aufschluß darüber geben könnte, ob die Schauer des 2. und 3. Maximums aus geladenen (direkt ionisierenden) oder neutralen (nur indirekt ionisierenden) Teilchen, oder beiden bestehen.

a) Einen Anhalt hierfür gewinnt man, wenn man die Ansprechwahrscheinlichkeit $w$ der Zählrohre auf die Schauerstrahlen untersucht. Für geladene Strahlen ist $w=1$. Für neutrale Strahlen muß $w<1$, wahrscheinlich sogar $w \ll 1$ sein. Zwei in der Strahlrichtung hintereinander angeordnete und parallel verbundene Zählrohre müssen daher für geladene Teilchen dieselbe, für ungeladene jedoch eine um den Faktor $(2-w)$ größere Ansprechwahrscheinlichkeit haben als ein einzelnes Zählrohr. Aus dieser Überlegung heraus wurde der folgende Versuch gemacht (Abb. 11). 16 Messingzählrohre von wiederum $60 \mathrm{~cm}$ Länge und $1 \mathrm{~mm}$ Wandstärke, aber nur $2 \mathrm{~cm}$ Durchmesser, wurden in zwei parallelen Lagen von je 8 untereinander an die Stelle der früheren gekreuzten Zählrohrlagen I+ II gebracht. Von den 8 oberen Zählrohren wurden je 2, durch ein Zählrohr und $1 \mathrm{~cm}$ Blei getrennte, in Zweifach-Koinzidenz geschaltet. Die unteren 8 Zählrohre blieben entweder außer Betrieb, oder sie wurden einzeln mit dem jeweils darüberliegenden Zählrohr parallelgeschaltet. Die erhaltenen Kurven zeigt Abb. 11. Wie man sieht, bewirkt das Zuschalten der unteren Zählrohre, daß das 2. Maximum etwa 2,4-mal so hoch wird, während das 3. Maximum nur wenig erhöht wird. Hierbei ist zu beachten, daß eine geringe Zunahme der Koinzidenzhäufigkeit auch bei ionisierenden Strahlen schon zu erwarten wäre, weil für die schräg verlaufenden Strahlen die Zählfläche etwas vergrößert wird. Aber selbst wenn man dies berücksichtigt, zeigt der Versuch, daß zumindest die Schauer des 2. Maximums neutrale Teilchen enthalten müssen.

Allgemeiner kann man folgende grobe Überlegung anstellen. Ein Schauer bestehe aus $n_{\mathrm{e}}$ geladenen und $n_{0}$ neutralen Teilchen. Für die letzteren sei $w \ll 1$ die Ansprechwahrscheinlichkeit eines Zählrohres. Trifft ein geladenes oder neutrales Schauerteilchen eines der oberen Zählrohre, so sei die Wahrscheinlichkeit $p$, daß ein weiteres geladenes oder neutrales Teilchen desselben Schauers das andere, in Koinzidenz ge-

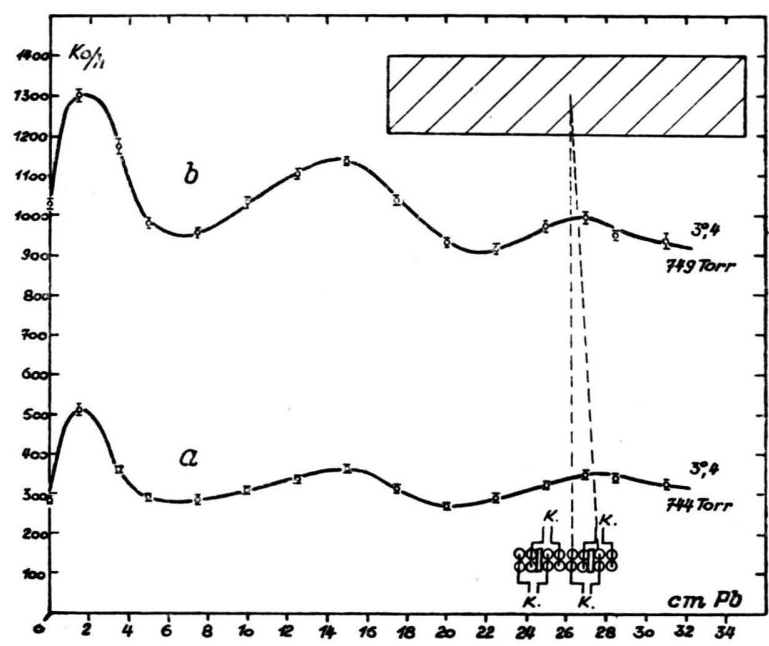

Abb. 11. Auslösekurven mit einfacher (a) und doppelter (b) Zählrohrlage.

schaltete Zählrohr trifft, stets dieselbe und $\ll 1$. Dann ist das Verhältnis der beobachteten Koinzidenzen mit und ohne untere Zählrohre

$R=\frac{n_{\mathrm{e}}\left(n_{\mathrm{e}}-1\right)+2 n_{\mathrm{e}} n_{0} w+4 n_{0}\left(n_{0}-1\right) w^{2}}{n_{\mathrm{e}}\left(n_{\mathrm{e}}-1\right)+n_{\mathrm{e}} n_{0} w+n_{0}\left(n_{0}-1\right) w^{2}}$.

Im Zähler wie im Nenner bezieht sich das 1., 2. und 3. Glied auf Koinzidenzen durch 2 geladene, bzw. 1 geladenes +1 neutrales, bzw. 2 neutrale Teilchen. Dies gilt streng, solange $n_{\mathrm{e}} p$ und $n_{0} w p \ll 1$ sind, aber qualitativ auch noch, wenn diese Größen $\approx 1$ sind.

Ist z. B. $n_{\mathrm{e}}=1$, so wird

$$
R=\frac{2+4\left(n_{0}-1\right) w}{1+\left(n_{0}-1\right) w} .
$$

Es muß natürlich mindestens $n_{0}=1$ sein. Aber der Wert $R \approx 2,4$ für das 2 . Maximum ist auch verträg- 
lich mit einem ziemlich großen $n_{0}$, wenn $w$ so klein ist, daß $n_{0} w$ von der Größenordnung 1/2 ist. Ein so hoher Wert von $n_{0} w$ wird sogar gefordert durch die relativ große Häufigkeit der Dreifach-Koinzidenzen (Ziff. 12). Die Versuchsergebnisse sind also sämtlich vereinbar mit der Annahme, daß die Schauer des 2. Maximums zusammengesetzt sind aus dem geladenen Primärteilchen und einer größeren Zahl von neutralen Teilchen, die von diesem ausgelöst wurden.

Werte $n_{\mathrm{e}}>1$ würden zwar ebenfalls nicht im Widerspruch zu unseren Versuchsergebnissen stehen, es müßte nur auch $n_{0}$ noch größer angenommen werden. Aber die Ergebnisse von Nebelkammerversuchen scheinen schwer mit $n_{\mathrm{e}}>1$ vereinbar (Ziff. 14).

Mit $n_{\mathrm{e}}=0$ andererseits würde die obige Gl. (3) $\boldsymbol{R}=4$ ergeben, was sicher zu hoch ist. Ein Wert $E \approx 2,4$ mit lauter neutralen Teilchen wäre nur möglich, wenn entweder die Ansprechwahrscheinlichkeit $w$ nicht viel kleiner als 1 , oder $n_{0}$ unwahrscheinlich groß wäre (es müßte $n_{0} w p \approx 1$ sein).

Ein Wert $R \approx 1$, wie für die Schauer des 3 . Maximums beobachtet, wäre nach $\mathrm{Gl}$. (3) nur möglich mit $n_{\mathrm{e}} \geq 2$. Ganz ist zwar $n_{\mathrm{e}}=0$ oder 1 nicht auszuschließen, aber dann müßte, entgegen unserer Voraussetzung, $n_{0} w p$ wesentlich $>1$ sein, was wiederum wenig wahrscheinlich ist. Andererseits ist aber auch $n_{\mathrm{e}}>2$ nicht sehr wahrscheinlich, wegen der relativ geringen Häufigkeit der Dreifach-Koinzidenzen (Ziff. 12). Die Schauer des 3. Maximums enthalten also wahrscheinlich zwei geladene Teilchen; daneben können sie auch eine gewisse Zahl $n_{0}$ von neutralen Teilchen enthalten, doch muß $n_{0} w$ wesentlich kleiner als 1 sein, damit der Wert $R=1$ nicht zu sehr überschritten wird. Hierfür spricht auch wiederum die vergleichsweise geringe Häufigkeit der Dreifach-Koinzidenzen (Ziff. 12).

Genauere Rechnungen über diese Fragen erscheinen vorläufig nicht lohnend.

b) An dieser Stelle sei noch folgender Versuch erwähnt. Die Zählrohre III wurden in Antikoinzidenz zu I + II geschaltet. Unter III befanden sich konstant $5 \mathrm{~cm}$ Blei, darüber variable Bleischichten von 0 bis $30 \mathrm{~cm}$ (umgekehrt wie bei dem Versuch Ziff. 9d). Die so gemessene Kurve verlief völlig horizontal, Maxima waren weder an den ursprünglichen Stellen des 2. und 3. Maximums, noch an anderen Stellen zu erkennen. Dieser Versuch bestätigt, daß die in der oberen Bleischicht entstehenden Schauer des 2. und 3. Maximums mindestens ein geladenes Teilchen (oder eine unwahrscheinlich große Zahl neutraler Teilchen) enthalten müssen.

\section{D is kussion}

Die Gesamtheit der beschriebenen Versuche reicht noch nicht aus, um die Prozesse, die zum Auftreten des 2. und 3. Maximums führen, vollständig zu klären und die beteiligten Teilchen eindeutig zu identifizieren. Doch gewähren diese Versuche wichtige Einblicke in die Genetik der Schauer des 2. und 3. Maximums.

Der auffallendste Zug am 2. und 3. Maximum ist ihre Schärfe. Diese wäre unverständlich, wenn es sich um eine direkte Umwandlung des Primärteilchens in einen Schauer handelte (Abb.12a), denn die Auslösekurve solcher Schauer wäre vom Typ $x \exp (-\alpha x)$

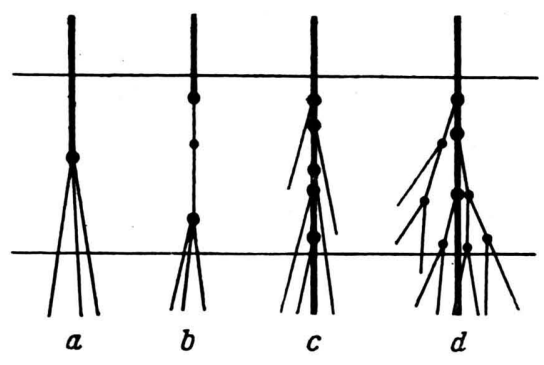

Abb. 12. Verschiedene Arten der direkten und indirekten Schauererzeugung (schematisch).

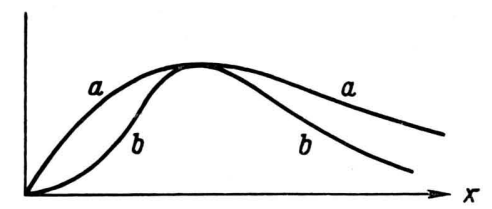

Abb. 13. Schematische Auslösekurven für direkte (a) und indirekte (b) Schauererzeugung.

$(x=$ Bleidicke, Abb. 13a); „Typ“ soll dabei heißen, daß der Übersichtlichkeit wegen die Absorbierbarkeit von Primär- und Sekundärstrahlen gleich angenommen wird. Das Maximum dieser Kurve ist zu flach. Vielmehr muß die Auslösekurve von dem Typ Abb. 13b sein, den man etwa beschreiben kann durch eine Funktion $x^{n} \exp (-\alpha x)$ mit $n>1$. Eine solche Kurve kann auf verschiedene Weise zustande kommen:

a) Ein Schauer entsteht zwar als Ganzes auf einmal, aber nicht unmittelbar aus der Primärstrahlung, sondern auf dem Wege über $n$ einfache Zwischenstrahlungen (Abb. 12b). Dann ist eine Kurve vom . Typ $x^{n+1} \exp (-\alpha x)$ zu erwarten. Die Rolle der „Zwischenstrahlung“ kann natürlich auch von der Primärstrahlung selbst übernommen werden, indem sie ihre Qualität oder ihre Energie ändert.

b) Das Primärteilchen erzeugt auf seinem Wege fortlaufend einzelne Sekundäre, die in ihrer Gesamt- 
heit den Schauer bilden (Abb. 12 c). Die Zahl der austretenden Sekundären ist dann vom Typ $x \exp (-\alpha x)$, ebenso auch die Häufigkeit der Koinzidenzen zwischen dem Primären und einem Sekundären. Dagegen ist offenbar die Häufigkeit der Koinzidenzen zwischen zwei Sekundären ungefähr vom Typ $x^{2} \exp (-\alpha x)$.

c) Die unter b) angenommenen Sekundären erzeugen noch Kaskadenschauer (Abb. 12d). Für einen isolierten solchen Schauer wird die Auslösekurve etwa vom Typ $x^{n} \exp (-\alpha x)$ sein, mit $n \geq 1$. Die resultierende Auslösekurve wird dann etwa vom Typ $x^{n+2} \exp (-\alpha x)$ sein.

d) Weitere Möglichkeiten ergeben sich, wenn man annimmt, daß instabile Teilchen mit sehr kurzer Lebensdauer im Spiel sind. Von solchen Deutungsmöglichkeiten wird man zunächst nur möglichst sparsam Gebrauch machen. Ausgeschlossen ist dabei jedenfalls eine direkte Schauerbildung durch ein zur Ruhe gekommenes Teilchen, weil die beobachteten Schauer dafür zu eng sind.

Wir mustern nun aus diesen Gesichtspunkten das experimentelle Material über die Schauer des 2. Max. Nach Ziff. 7 und 10 werden sie durch geladene Teilchen ausgelöst, deren Durchdringungsvermögen so groß ist, daß es nicht Elektronen sein können. Für die Frage nach den auslösenden Teilchen ist weiter die absolute Häufigkeit der beobachteten Schauer von Wichtigkeit. Diese wurde in Ziff. $6 \mathrm{zu} \mathrm{rd.} 2 / \mathrm{cm}^{2} \cdot \mathrm{h}$ ermittelt unter der Voraussetzung, daß die Schauer aus zwei geladenen Teilchen bestehen. Die Zahl der $\mu$-Mesonen in dem Raumwinkel, den der Schauerstrahler den Zählrohren I + II darbietet, ist ${ }^{36}$ rd. $8 / \mathrm{cm}^{2} \cdot \mathrm{h}$, d. h. ein Schauer kommt auf rd. $4 \mu$-Mesonen.

Nach Ziff. 13 ist es jedoch viel wahrscheinlicher, daß die Schauer des 2. Maximums nur 1 geladenes und eine gewisse Zahl $n_{0}$ von neutralen Teilchen mit der Ansprechwahrscheinlichkeit $w \ll 1$ enthalten. In der Tat sprechen die von anderer Seite angestellten Nebelkammerversuche ${ }^{38-45}$, soweit sie einen Vergleich mit unseren Zählrohrversuchen zulassen, in ihrer Gesamtheit dafür, daß die mittlere Zahl der geladenen Teilchen in den Schauern des 2. Maximums nicht wesentlich größer als 1 sein kann: enge Paare ionisie-

38 H. M a i e r - L e i b n it z, Z. Physik 112, 569 [1939].

39 R. P. Shutt, Physic. Rev. 69, 261 [1946].

40 L. S e r e n, Physic. Rev. 62, 204 [1942].

41 S. L e is e g a n g, Z. Physik 116, 515 [1940].

42 W. H. B o s ti c k, Physic. Rev. 61, 557 [1942].

43 E. D. W o ll a n, Physic. Rev. 60,532 [1941].

44 M. P o w e 11, Physic. Rev. 60, 413 [1941].

45 D. J. H u ghe s, Physic. Rev. 60, 414 [1941]. render Teilchen aus Blei wurden zwar beobachtet, aber ihre Häufigkeit relativ zu der der $\mu$-Mesonen bleibt, bei Berücksichtigung, aller geometrischen Faktoren, um etwa eine Größenordnung hinter der hier gemessenen Schauerhäufigkéit zurück ${ }^{46}$. Nimmt man also nur ein geladenes Teilchen an, und ist $n_{0} w<1$, so muß die wirkliche Häufigkeit der Schauer sogar noch größer sein als oben angegeben. Jedenfalls wird aber $n_{0} w$ nicht $\gg 1$ sein. Daher ist das Häufigkeitsverhältnis Schauer: $\mu$-Mesonen mindestens von der Größenordnung 1/10. Dies läßt es ziemlich ausgeschlossen erscheinen, daß die auslösenden Teilchen $\pi$-Mesonen oder Protonen sind. Die $\pi$-Mesonen und die primären Protonen ${ }^{47}$ sind im Meeresniveau zu selten, die sekundären, aus Kernzertrümmerungen (,Sternen“) stammenden Protonen haben zu kleine Energie. Es kommen also nur noch die $\mu$-Mesonen in Betracht, und zwar scheinen diese als durchgehende Bestandteile der Schauer während ihres ganzen Aufbaues aufzutreten. Dies zeigen die Versuche Ziff. $9 \mathrm{~d}$ und Ziff. $13 \mathrm{~b}$, bei denen AntikoinzidenzZählrohre in den Schauerstrahler eingebaut waren; das Verschwinden des 2. Maximums ist so zu deuten, daß die Schauer während ihrer vertikalen Entwicklung ständig ein ionisierendes Teilchen enthalten müssen. Hiernach und auf Grund von Ziff. $13 \mathrm{a}$ ist es am wahrscheinlichsten, daß die Schauer des 2. Maximums aus den gewöhnlichen $\mu$-Mesonen und einer größeren Zahl von größtenteils neutralen Sekundärteilchen bestehen, die von den Mesonen beim Durchgang durch Materie fortlaufend erzeugt werden und die möglicherweise noch kaskadenartige Vervielfachung erfahren ${ }^{48}$.

Hier taucht natürlich der Verdacht auf, daß es sich einfach um Elektronenkaskaden handelt, die aus den Stoßelektronen der $\mu$-Mesonen entstehen. Hiergegen sprechen wiederum die folgenden, schon früher ${ }^{3}$ aufgeführten Argumente ${ }^{50}$.

46 Dagegen könnten vielleicht die mehrfach festgestellten "anomalen Streuungen“ von Mesonen durch die Schauer des 2. Maximums erklärt werden.

47 A. Vanna, G. Co c coni u. V. Tongiorgi, Physic. Rev. 76, 517 [1949].

48 Fortlaufende Erzeugung von Sekundären durch die Mesonen hat schon Ehme rt ${ }^{49}$ zur Deutung der engen Schauer vermutet, allerdings wurden die Sekundärteilchen als geladen angenommen, was nach Obigem kaum möglich ist.

49 A. E h m ert, Z. Physik 113, 234 [1939].

50 Dagegen könnte vielleicht das kürzlich von $\mathrm{M}$ a $\mathrm{z}$ e 51 berichtete Nebenmaximum bei $6 \mathrm{~cm}$ Blei auf diese Weise seine Erklärung finden. (Anm. b. d. Korr.: Wir haben inzwischen dieses Nebenmaximum gesucht, aber nicht finden können.) 
Die auf diese Weise entstehende Auslösekurve wäre ungefähr (nämlich abgesehen von der Absorption der auslösenden Mesonen) die Integralkurve der Auslösekurve eines einzelnen Elektronenschauers. Das 2. Maximum müßte’ also spätestens bei derjenigen Bleidicke eintreten, bei der die „Reichweite“ eines einzelnen Schauers liegt, in Wirklichkeit sogar wesentlich früher wegen der Absorption der auslösenden Mesonen. Die Lage des 2. Maximums bei $16 \mathrm{~cm}$ Blei dürfte kaum mit der bekannten kurzen Reichweite der Elektronenschauer vereinbar sein, zumal die Stoßelektronen größtenteils nur kleine Energie haben.

Ferner spricht auch das große Durchdringungsvermögen der Schauer (Ziff. 11) gegen ihre ElektronPhoton-Natur. Dieses Argument dürfte auch noch in Kraft bleiben, wenn man annimmt, daß das eine der beiden koinzidierenden Schauerteilchen das auslösende $\mu$-Meson selbst ist und daher ein sehr großes Durchdringungsvermögen hat.

Schließlich haben auch die Schauer des 2. Maximums eine wesentlich kleinere Winkeldivergenz als die Elektronenkaskaden (Ziff. 6).

Die Frage, welcher Art die neutralen Schauerteilchen des 2. Maximums sind, muß also noch offenbleiben.

Bezüglich des 3. Maximums haben die Versuche ergeben, daß es durch eine neutrale Strahlung erzeugt wird (Ziff. $7 \mathrm{c}$ ), deren vertikaler Intensitätsverlauf demjenigen der $\mu$-Mesonen wenigstens grob proportional ist (Ziff. 10). Die Häufigkeit der beobachteten Schauer, die wahrscheinlich 2 geladene, aber möglicherweise auch eine gewisse Zahl neutraler Teilchen enthalten (Ziff. 13), ist von der Größenordnung $1 / \mathrm{cm}^{2} \cdot \mathrm{h}$ (Ziff. 6). Nach der oben über das 2. Maximum angestellten Überlegung muß daher die Intensität der auslösenden neutralen Strahlen nahezu von derselben Größenordnung wie die der $\mu$-Mesonen sein. Daß dies Photonen sind, die etwa von den $\mu$ Mesonen fortlaufend erzeugt werden, erscheint wenig plausibel, weil dann überwiegend die gewöhnlichen Elektronenkaskaden zu erwarten wären ${ }^{52}$. Auch Neutronen können als auslösende Teilchen schwerlich in Betracht kommen, denn die meisten im Meeresniveau vorkommenden Neutronen, die aus „Sternen“ stammen, haben viel zu geringe Energie $(<50 \mathrm{MeV})$, und

51 R. M a ze u. Ts a i-Chü, C. R. hebd. Séances Acad. Sci. 332, 224 [1951], zitiert nach Physics Abstr. Nr. 2795, 54, 322 [1951].

52 Erst bei Photonenenergien oberhalb etwa $10^{14} \mathrm{eV}$ sollte die Erzeugung von Mesonenpaaren mit der Erzeugung von Elektronenpaaren konkurrieren können 53,54 die energiereichen Neutronen sind zu selten ${ }^{47}$. Unter diesen Umständen muß man wohl ernstlich an die Möglichkeit denken, daß neutrale $\mu$-Mesonen für die Schauer des 3. Maximums verantwortlich sind (neutrale $\pi$-Mesonen scheiden wegen ihrer kurzen Lebensdauer von $<5 \cdot 10^{-14} \mathrm{sec}$ aus ${ }^{55}$ ). Neutrale $\mu$-Mesonen können aus geladenen durch Ladungsaustausch entstehen. Leider ist über das neutrale $\mu$-Meson noch $\mathrm{zu}$ wenig bekannt, um diese Möglichkeit im einzelnen zu diskutieren.

Beim 3. Maximum ist die große Schärfe noch auffälliger als beim 2. Maximum. Die Auslösekurve dieser Schauer muß also noch ausgeprägter vom Typ Abb. 13b sein, d. h. die Auslösung muß ein stufenweiser Prozeß nach Art der oben mit a) bis c) bezeichneten sein. Einen direkten Beweis hierfür liefert der Versuch Ziff. 9d, welcher zeigte, daß die obersten $5 \mathrm{~cm}$ des Bleistrahlers nur einen sehr geringen Beitrag zur Gesamtzahl der im 3. Maximum beobachteten Schauer liefern (vgl. die nähere Diskussion im Anhang). Derselbe Versuch lehrt noch, daß diese Schauer auch in den Zwischenstadien ihrer Entwicklung noch wesentlich aus neutralen Teilchen bestehen müssen, andernfalls müßte bei Einbringen der Antikoinzidenzzählrohre unter die obersten $5 \mathrm{~cm}$ Blei das 3. Maximum um $5 \mathrm{~cm}$ nach rechts rücken.

$\mathrm{Da}$ es sich auch hier nicht um gewöhnliche Elektronenkaskaden handeln kann, die etwa durch die auslösende Strahlung eingeleitet werden, dafür gelten die schon oben für das 2. Maximum aufgeführten Argumente in verstärktem Maße: ein Maximum bei $26 \mathrm{~cm}$ Blei (50 Strahlungslängen!) kann unmöglich auf diese Weise erklärt werden.

Wenn die Schauer des 3. Maximums zwei geladene Mesonen enthalten, sollten sie sich in der Nebelkammer unter geeigneten Bedingungen zeigen. Erzeugung eines engen Mesonenpaares durch ein neutrales Teilchen ist von $\mathrm{Pow}$ e $11^{56}$ beobachtet worden. Es liegen jedoch zu wenig Nebelkammerversuche mit geeigneten Bleifiltern vor, um einen Häufigkeitsvergleich anstellen zu können.

Der merkwürdigste Punkt ist wohl die Abhängigkeit der Lage des 3. Maximums von äußeren Bedingungen. Feste Materieschichten, die in einigen Metern Höhe über dem Schauerstrahler angebracht werden.

53 F. B ooth u. A. H. Wilson, Proc. Roy. Soc. [London], Ser. A 175, 483 [1940].

54 R. F. C risty u. S. K us aka, Physic. Rev. 59, 405 [1941].

55 C. F. P ow e ll, Rep. Progr. Physics 13, 350 [1950].

56 M. P ow ell, Physic. Rev. 58, 474 [1940] u. 69, $385\lceil 1946\rceil$. 
ändern die Lage des 3. Maximums nicht (Ziff. 9). Auch in $2300 \mathrm{~m}$ Höhe liegt das 3. Maximum praktisch an derselben Stelle wie im Meeresniveau. Dagegen hängt die Lage des 3. Maximums in anscheinend streng gesetzmäßiger Weise von dem auf Meeresniveau reduzierten Luftdruck ab (Ziff. 8). Mit dem Zustand der höheren Atmosphäre besteht eine Korrelation nur so weit, wie sie zwischen diesem Zustand und dem reduzierten Barometerstand vorhanden ist. Über diesen Sachverhalt kann man sich verschiedene Gedanken machen, doch möchten wir mit einer eingehenden Diskussion noch zurückhalten, bis mehr experimentelles Material vorliegt. Vermutlich wird man nicht ohne Heranziehung instabiler Teilchen von kurzer Lebensdauer auskommen.

Hiermit zusammenhängend seien noch einmal kurz die beiden Versuche Ziff. $9 \mathrm{c}$ und d gegenübergestellt. Ein 5-cm-Bleifilter, $1 \mathrm{~m}$ über dem mit AntikoinzidenzZählrohren bedeckten Schauerstrahler, hat auf die Lage des 3. Maximums wenig Einfluß, während dasselbe Filter, unmittelbar auf die Antikoinzidenzzählrohre gelegt, das 3. Maximum um fast die ganze Filterdicke verschiebt. Es ist nicht ausgeschlossen, daß dieser Unterschied triviale geometrische Gründe hat, obwohl dies bei der Enge der Schauer nicht ganz leicht zu verstehen wäre. Eine andere Deutung würde darin bestehen, daß in irgendeinem Entwicklungsstadium der Schauer ein instabiles Teilchen mit einer Lebensstrecke von rd. $1 \mathrm{~m}$, also einer Lebensdauer von der Größenordnung $10^{-8}$ sec auftritt. Diese Annahme erfordert aber noch deutlichere Beweise.

Weitere Klärung der in dieser Arbeit behandelten Fragen wird von Nebelkammerversuchen erhofft, die gegenwärtig im Anlaufen sind.

Bei dieser Untersuchung wurden Mittel mitbenutzt, die die Notgemeinschaft der Deutschen Wis senschaft dem einen von uns (B.) dankenswerterweise zur Verfügung gestellt hat.

\section{Anhang:}

Diskussion des Versuches Ziff. $9 d$

Wir legen den einfachsten der in Ziff. 14 diskutierten Auslöseformalismen für die Schauer des 3. Maximums zugrunde: die neutrale Primärstrahlung erzeugt eine neutrale Zwischenstrahlung, die ihrerseits die ionisierenden Schauer erzeugt. Alle drei Strahlungen mögen denselben Absorptionskoeffizienten $a$ haben. Auf kompliziertere Fälle lassen sich die Überlegungen leicht übertragen. Die gesamte Bleidicke sei $x$, wovon der Teil $a$ über den Antikoinzidenz-Zählrohren liege. $x_{1}$ und $x_{2}$ seien die Tiefen, in denen die beiden Strahlenumwandlungen erfolgen. Ohne Antikoinzidenz-Zählrohre wird dann die beobachtete Schauerhäufigkeit, bis auf einen belanglosen Faktor,

$$
\begin{array}{r}
N=\int_{0}^{x} e^{-a x_{1}} \mathrm{~d} x_{1} \int_{x_{1}}^{x} e^{-a\left(x_{2}-x_{1}\right)} \mathrm{d} x_{2} e^{-a\left(x-x_{2}\right)} \\
=\frac{1}{2} x^{2} e^{-a x} .
\end{array}
$$

Dieser Ausdruck hat sein Maximum bei $x_{m}=2 / \alpha$ $=26 \mathrm{~cm}$, somit $1 / \alpha=13 \mathrm{~cm}$. Die Höhe des Maximums beträgt $N_{m}=45,7$. Führt man nun in der Tiefe $a=5 \mathrm{~cm}$ die Antikoinzidenz-Zählrohre ein, so wird entsprechend

$$
\begin{array}{r}
N=e^{-a x}\left(\int_{0}^{a} \mathrm{~d} x_{1} \int_{a}^{x} \mathrm{~d} x_{2}+\int_{a}^{x} \mathrm{~d} x_{1} \int_{x_{1}}^{x} \mathrm{~d} x_{2}\right) \\
=\frac{1}{2}\left(x^{2}-a^{2}\right) e^{-a x} .
\end{array}
$$

Jetzt wird

$$
\begin{gathered}
x_{m}=\frac{1}{a}+\sqrt{\frac{1}{a^{2}}+a^{2}}=26,9 \mathrm{~cm}, \\
N_{m}=44,2 .
\end{gathered}
$$

Das 3. Maximum sollte also in der Tat nur wenig niedriger, aber um $0,9 \mathrm{~cm}$ nach rechts verschoben sein. Die beobachtete Verschiebung beträgt sogar nur $5-4,5=0,5 \mathrm{~cm}$. Die Geringfügigkeit der Verschiebung erklärt sich hiernach daraus, daß über den Antikoinzidenz-Zählrohren schon die Zwischenstrahlung sich aufbauen kann, während die fertigen Schauer, die dort entstehen und durch Antikoinzidenzen unwirksam gemacht werden, nur einen kleinen Bruchteil der maximalen Schauerproduktion ausmachen.

Ohne die Zwischenstrahlung dagegen müßte offenbar das Maximum um die vollen $5 \mathrm{~cm}$ verschoben sein, weil das Blei über den Antikoinzidenz-Zählrohren vollständig unwirksam wäre. Der Versuch beweist also die stufenweise Entstehung der Schauer. 